\title{
Digestible indispensable amino acid scores of nine cooked cereal grains
}

\author{
Fei $\operatorname{Han}^{1}{ }^{1} \dagger$, Fenli $\operatorname{Han}^{1,2} \dagger$, Yong Wang ${ }^{1} \dagger$, Liuping Fan ${ }^{2}$, Ge Song ${ }^{1}$, Xi Chen ${ }^{1}$, Ping Jiang ${ }^{1}$, Haijiang Miao ${ }^{1}$ \\ and Yangyang $\operatorname{Han}^{1,2}$ \\ ${ }^{1}$ Institution of Grain Quality and Nutrition, Academy of State Administration of Grain, Beijing 100037, People's Republic of China \\ ${ }^{2}$ School of Food Science and Technology, Jiangnan University, Wuxi, Jiangsu 214122, People's Republic of China
}

(Submitted 27 March 2018 - Final revision received 27 August 2018 - Accepted 21 September 2018 - First published online 6 November 2018)

\begin{abstract}
True ileal digestibility (TID) values of amino acid (AA) obtained using growing rats are often used for the characterisation of protein quality in different foods and acquisition of digestible indispensable amino acid scores (DIAAS) in adult humans. Here, we conducted an experiment to determine the TID values of AA obtained from nine cooked cereal grains (brown rice, polished rice, buckwheat, oats, proso millet, foxtail millet, tartary buckwheat, adlay and whole wheat) fed to growing Sprague-Dawley male rats. All rats were fed a standard basal diet for $7 \mathrm{~d}$ and then received each diet for $7 \mathrm{~d}$. Ileal contents were collected from the terminal $20 \mathrm{~cm}$ of ileum. Among the TID values obtained, whole wheat had the highest values $(P<0.05)$, and polished rice, proso millet and tartary buckwheat had relatively low values. The TID indispensable AA concentrations in whole wheat were greater than those of brown rice or polished rice $(P<0 \cdot 05)$, and polished rice was the lowest total TID concentrations among the other cereal grains. The DIAAS was 68 for buckwheat, 47 for tartary buckwheat, 43 for oats, 42 for brown rice, 37 for polished rice, 20 for whole wheat, 13 for adlay, 10 for foxtail millet and 7 for proso millet. In this study, the TID values of the nine cooked cereal grains commonly consumed in China were used for the creation of a DIAAS database and thus gained public health outcomes.
\end{abstract}

Key words: Cooked cereal grains: Digestible indispensable amino acid scores: True ileal digestibility: Protein quality: Growing rats

Accurately estimating the dietary protein and amino acid (AA) digestibility of food products is necessary ${ }^{(1)}$. The protein digestibility-corrected amino acid score (PDCAAS) has been adopted by the Joint FAO/WHO Expert Consultation since 1991 and has since been used for the evaluation of protein quality in food products ${ }^{(2)}$. However, this method has several limitations ${ }^{(3,4)}$. The main difference between the newly recommended digestible indispensable AA score (DIAAS) and PDCAAS is that the true ileal AA digestibility for the dietary indispensable AA is used in DIAAS rather than a single faecal crude protein $(\mathrm{CP})$ digestibility value. AA are absorbed from the small intestine only and are metabolised extensively by the microbiota of the hindgut. Terminal ileal digestibility is more accurate than faecal digestibility in estimating AA bioavailability ${ }^{(5-8)}$. Moreover, PDCAAS underestimates the comparatively high nutritional values of some proteins by truncation and overestimates the quality of proteins containing antinutritional factors and limiting $\mathrm{AA}^{(9-14)}$. In contrast, DIAAS is not truncated for a single-source protein and is preferred to PDCAAS for the evaluation of protein quality by the $\mathrm{FAO}^{(15)}$. Digestibility should be based on the true ileal digestibility (TID) of each AA, which is preferably determined in humans, but if this is not possible, TID can be determined in growing pigs or rats ${ }^{(16)}$

Cereal grains are often the main component of the human diet and provide a large proportion of the dietary protein for humans, especially in developing countries ${ }^{(17)}$. Thus, accurately assessing the protein nutritional value of cereal grains is essential ${ }^{(11)}$. Cereal grains and grain by-products are usually cooked before human consumption. Directly determining ileal AA digestibility in humans is difficult and expensive; thus, the Expert Consultation (FAO, 2013) recommended the use of pigs, which are the best models for adult humans; alternatively, growing rat can also be used $^{(15,18,19)}$. In this study, we aimed to determine the apparent ileal digestibility (AID), TID values of AA and DIAAS values in nine cooked cereal grains fed to growing rats.

\section{Methods}

Materials

Brown rice, polished white rice, oats, tartary buckwheat, buckwheat, foxtail millet, proso millet, adlay and wheat

Abbreviations: AA, amino acid; AID, apparent ileal digestibility; CP, crude protein; DIAAS, digestible indispensable amino acid score; PDCAAS, protein digestibility-corrected amino acid score; TID, true ileal digestibility.

* Corresponding author: F. Han, email hf@chinagrain.org

$\dagger$ These authors contributed equally to this work. 
were used. Adlay was purchased from the Guizhou Province, while oats and foxtail millet were procured from Inner Mongolia. The other cereal grains were obtained from Northwest A\&F University. Wheat was baked into wheat bread according to the national standard (LST 3204-1993). The other cereal grains were soaked for $30 \mathrm{~min}$ with $25^{\circ} \mathrm{C}$ deionised water. The cereals were then cooked using a commercially available cooker as described by the manufacturer. The respective proportions of brown rice, polished white rice, oats, tartary buckwheat, buckwheat, foxtail millet, proso millet or adlay to water were $1: 1 \cdot 6,1: 1 \cdot 6,1: 2 \cdot 3,1: 20,1: 20,1: 1 \cdot 8,1: 1 \cdot 9$ or $1: 1 \cdot 4$ $(\mathrm{w} / \mathrm{v})$, respectively. All the cooked materials were freeze dried after cooking, and all the materials were ground through a size60 mesh before inclusion into the diets.

\section{Animals and diets}

The animal experiments used 150 male Sprague-Dawley rats that were approximately $240 \mathrm{~g}$ in weight and were purchased from the Beijing Vital River Laboratory Animal Center. Rats were caged individually and were maintained under controlled temperature $\left(22 \pm 2^{\circ} \mathrm{C}\right)$, humidity and airflow condition, with a 12-h on-off light cycle as described by Rutherfurd et al. ${ }^{(3)}$. Adequate measures were taken to minimise the pain or discomfort of the rats, and we used the smallest possible number of animals. The study was reviewed and approved by the Institutional Animal Ethics Committee at Jiangnan University (JN. No. 20170930k1201105 [36]). All animals were maintained according to local regulations and guidelines.

A total of eleven semisynthetic wheat starch-based diets (Table 1) were formulated to contain $100 \mathrm{~g} / \mathrm{kg} \mathrm{CP}$, which was the sole protein source. To meet the requirements for growing rats, we added vitamins and minerals. A total of $3 \mathrm{~g} / \mathrm{kg}$ of titanium dioxide was included in each diet as an indigestible marker. Purified sucrose, soyabean oil and cellulose were mixed in a ratio of 10:5:3 ( $180 \mathrm{~g} / \mathrm{kg} \mathrm{DM})$. To maintain a dietary $\mathrm{CP}$ concentration of $100 \mathrm{~g} / \mathrm{kg}$ for low-protein foods with $\mathrm{CP}$ concentration of $<150 \mathrm{~g} / \mathrm{kg} \mathrm{DM}$, the test ingredient was diluted with cellulose and soyabean oil (1:0·6); for foods with $\mathrm{CP}$ concentration of $<100 \mathrm{~g} / \mathrm{kg} \mathrm{DM}$, the test diet consists of the test ingredient, vitamin/mineral mixture and titanium dioxide $^{(20)}$. The ingredient compositions of the basal and test diets are shown in Table 1. A protein-free-based diet was prepared for rats to determine the amount of endogenous loss of AA in the ileal content ${ }^{(21)}$. A basal diet containing $100 \mathrm{~g} / \mathrm{kg}$ protein was also formulated using casein as the sole source of protein $^{(22)}$.

\section{Experimental design}

The rats ( $n$ 150) were randomly divided into 10 groups ( $n$ 15/ group) as follows: brown rice group, polished rice group, buckwheat group, oats group, proso millet group, foxtail millet group, tartary buckwheat group, adlay group, whole wheat group and protein-free-based diet group. All rats were initially fed a standard basal diet for $7 \mathrm{~d}$. After 1 week of acclimatisation period, the experimental groups received each diet in Table 1 for $7 \mathrm{~d}$. Each rat received its respective diet in nine hourly meals (08.30-16.30 hours) daily. The diet was freely available for $10 \mathrm{~min}$ at each meal time. Water was also freely available. On the 14th day of the study, each rat was killed $5 \mathrm{~h}$ after the first meal through asphyxiation with $\mathrm{CO}_{2}$ gas ${ }^{(3,23)}$. Ileal contents were immediately collected from the terminal $20 \mathrm{~cm}$ of ileum. Given that the ileal content of each rat is insufficient for the AA detection through HPLC $^{(22)}$, three ileum contents were mixed into one sample in each group $(n 5)$. All ileal content samples were freeze-dried and frozen $\left(-20^{\circ} \mathrm{C}\right)$ while awaiting chemical analysis.

\section{Chemical analysis}

CP content was determined by rapid N cube (NY/T 20072011) using a N-to-protein conversion factor of $6 \cdot 25$. The AA contents were determined in triplicate 5-mg samples following hydrolysis in $500 \mu \mathrm{l}$ of constant-boiling $\mathrm{HCl}(6 \mathrm{~mol} / \mathrm{l})$ for $24 \mathrm{~h}$ at $110 \pm 1^{\circ} \mathrm{C}$ in a hydrolysis tube ${ }^{(24)}$. The liberated AA were derived with 6-aminoquinolyl- $N$-hydroxysuccinimidyl carbamate, and $\alpha$-aminobutyric acid was used as the internal standard. The derivatives were separated on a Waters E2695 HPLC system equipped with a C18 column $(150 \mathrm{~mm} \times 4.6 \mathrm{~mm}$, $5.0 \mu \mathrm{m}$; Agilent) and quantified using Waters 2475 fluorescence detector at $395 \mathrm{~nm}$ emission and $250 \mathrm{~nm}$ excitation. To determine cysteine and methionine, we used performic acid oxidation at $0^{\circ} \mathrm{C}$ for $16 \mathrm{~h}$, followed by neutralisation with $\mathrm{HBr}$; then, we applied hydrolysis as described above. The concentration of titanium in the diets and ileal samples was determined through the method described by Short et al. ${ }^{(25)}$. The samples were ashed, then digested in $60 \%(\mathrm{v} / \mathrm{v})$ sulfuric acid and finally added to $30 \%$ hydrogen peroxide. Absorbance at $410 \mathrm{~nm}$ was measured. Tryptophan (Trp) was determined using the method described by Rutherfurd \& Gilani ${ }^{(24)}$. Free AA molecular weights were used for the calculation of the weight of each AA.

\section{Data analysis}

AA and CP contents in the terminal ileal digesta and the TID of AA were calculated by using the equation given by Rutherfurd et $a l .{ }^{(26)}$. In addition, the endogenous ileal AA flows were determined for rats fed the protein-free $\operatorname{diet}^{(27)}$.

Apparent and true ileal AA digestibility was calculated using the following equations (units are $\mathrm{g} / \mathrm{kg}$ DM intake) ${ }^{(6,28)}$ :

$$
\mathrm{AID}_{\mathrm{AA}}=1-\left(\left(\mathrm{AA}_{\text {digesta }} / \mathrm{AA}_{\text {diet }}\right) \times\left(\mathrm{Ti}_{\text {diet }} / \mathrm{Ti}_{\text {digesta }}\right)\right) \times 100,
$$

where $\mathrm{AID}_{\mathrm{AA}}$ is the $\mathrm{AID}$ of $\mathrm{AA}(\%), \mathrm{AA}_{\text {digesta }}$ is the concentration of $\mathrm{AA}$ in the ileal digesta $\mathrm{DM}, \mathrm{AA}_{\text {diet }}$ is the concentration of $\mathrm{AA}$ in the diet $\mathrm{DM}, \mathrm{Ti}_{\text {diet }}$ is the concentration of $\mathrm{Ti}$ in the diet $\mathrm{DM}$ and $\mathrm{Ti}_{\text {digesta }}$ is the concentration of $\mathrm{Ti}$ in the ileal digesta DM.

$$
\mathrm{TID}_{\mathrm{AA}}=\mathrm{AID}+\left(\left(\mathrm{IAA}_{\mathrm{end}} / \mathrm{AA}_{\text {diet }}\right) \times 100\right),
$$

where $\mathrm{IAA}_{\text {end }}$ is the ileal endogenous AA losses. 
DIAA reference ratio $=\frac{\mathrm{mg} \text { of the digestible dietary indispensable } \mathrm{AA} \text { in } 1 \mathrm{~g} \text { of the test protein }}{\mathrm{mg} \text { of the dietary indispensable } \mathrm{AA} \text { in } 1 \mathrm{~g} \text { of the reference protein }}$

where the reference protein indispensable AA profile was the AA requirement pattern for the $0.5-3$ years old child ${ }^{(15)}$.

DIAAS was calculated using the following equation ${ }^{(15,20)}$ :

$\operatorname{DIAAS}(\%)=100 \times$ lowest value of the digestible indispensable

AA reference ratio.

\section{Statistical analysis}

Calculation of sample size was performed using the 'resource equation' method, as described by Charan \& Kantharia ${ }^{(29)}$, with a power of $80 \%$ and significance of $5 \%$. Results were expressed as mean values with their standard errors. The Shapiro-Wilk comparison normality test was used to assess the distribution of all variables. Comparisons for normally distributed data between the two groups were conducted using two-tailed $t$ test and one-way ANOVA followed by Tukey's significance test for multiple comparisons. Mann-Whitney $U$ and Kruskal-Wallis tests were used for non-parametric analysis when data were non-normally distributed. A $P$ value of $<0 \cdot 05$ was considered significant. All statistical calculations were performed on SPSS 21.0 data processing software (SPSS Inc.).

\section{Results}

Crude protein and amino acid compositions of nine cooked cereal grains

A total of eighteen AA were detected in nine cooked cereal grains. The total AA concentrations of the nine cooked cereal grains on an as-fed basis ranged from $8.3 \%$ (polished rice) to $18.5 \%$ (adlay; Table 2). The CP contents of the cooked cereal grains ranged from $9.15 \%$ (polished rice) to $19.28 \%$ (adlay). The CP contents of buckwheat, oats, proso millet, foxtail millet, adlay and whole wheat were higher $(P<0.05)$ than those of brown rice, polished rice and tartary buckwheat. The cooked cereal grains had indispensable AA contents, ranging from 30.5 (brown rice) to $66.3 \mathrm{~g} / \mathrm{kg}$ DM (adlay). The AA compositions in the diets based on the nine cooked cereal grains are shown in Table 3 .

\section{Mean apparent ileal digestibility of amino acids in nine} cooked cereal grains

The mean AID of indispensable AA in whole wheat was greater than that in any of the other cooked cereal grains (Table 4). The AID values of most AA in whole wheat were nonsignificantly different from those in adlay, except that the AID of leucine (Leu) in whole wheat was lower than that in adlay $(P<0.05)$, whereas the AID of lysine (Lys) in whole wheat was higher than that in adlay $(P<0.05)$. The mean AID of the indispensable AA and AID of Lys and Trp in proso-millet were the lowest among the values obtained for all cooked cereal grains. The mean AID of the indispensable AA and AID of all indispensable AA in proso millet were significantly lower $(P<0.05)$ than that in foxtail millet. The mean AID of all AA in adlay were all greater than those in all the other cooked cereal grains, except whole wheat. Meanwhile, the mean AID of all AA in polished rice and proso millet were lowest among the values obtained for all cooked cereal grains $(P<0 \cdot 05)$.

\section{Mean true ileal digestibility of amino acids in nine cooked cereal grains}

The mean TID of indispensable AA in whole wheat and adlay were greater than those for other cooked cereals $(P<0.05$; Table 5). Furthermore, no difference was observed in the mean TID of indispensable AA between adlay and whole wheat. No difference was observed between the indispensable AA TID values of buckwheat and foxtail millet, although Leu and Trp TID values were greater $(P<0.05)$ in foxtail millet than in buckwheat. The mean TID of the indispensable AA in polished rice, proso millet and tartary buckwheat were lower $(P<0.05)$ than those of the other cooked cereal grains.

\section{Mean true ileal digestibility concentrations for amino acids in nine cooked cereal grains}

The total TID concentrations of indispensable AA in buckwheat were significantly lower than those for adlay, foxtail millet, proso millet and oats and significantly greater than that for brown rice, tartary buckwheat and polished rice $(P<0.05$; Table 6$)$. Adlay had the highest TID concentrations of valine, isoleucine, Leu and tyrosine among the cooked cereal grains $(P<0.05)$, and buckwheat and brown rice had the highest TID concentrations of Lys and Trp, respectively $(P<0.05)$. Polished rice had the lowest total TID concentration of indispensable AA $(P<0.05)$.

\section{Digestible indispensable amino acid score for nine cooked cereal grains}

The following DIAAS values were obtained: 42 , brown rice; 37 , polished rice; 68 , buckwheat; 43 , oats; 7 , proso millet; 10 , foxtail millet; 47 , tartary buckwheat; 13 , adlay and 20 , whole wheat (Table 7).

\section{Discussion}

The nine cereal grains tested in this study are commonly produced in different provinces in China. Buckwheat and tartary buckwheat belonging to Polygonaceae family grow mainly in Russia, China and India ${ }^{(30)}$. Proso millet (Panicum miliaceum L.) is consumed as a staple food among the majority of people who live in arid and semi-arid tropics of the world, such as Asia, Africa and some parts of Europe ${ }^{(31)}$. Foxtail millet (Setaria italica) is one of the most important food crops of the semi-arid tropics in 
Table 1. Ingredient composition of the experimental diet ( $\mathrm{g} / \mathrm{kg} \mathrm{DM})$

\begin{tabular}{|c|c|c|c|c|c|c|c|c|c|c|c|}
\hline Composition & Brown rice & Polished rice & Buckwheat & Oats & Proso millet & Foxtail millet & Tartary buckwheat & Adlay & Whole wheat & Protein-free diet & Basal diet \\
\hline Wheat starch & - & - & 88.0 & $104 \cdot 0$ & 171.0 & - & - & 233.0 & $157 \cdot 0$ & 752.0 & 637.0 \\
\hline Soyabean oil & - & - & 50.0 & $50 \cdot 0$ & 50.0 & $96 \cdot 9$ & - & 50.0 & $50 \cdot 0$ & $50 \cdot 0$ & 50.0 \\
\hline Purified cellulose & - & - & 30.0 & $30 \cdot 0$ & 30.0 & $58 \cdot 1$ & - & $30 \cdot 0$ & 30.0 & 30.0 & 30.0 \\
\hline Purified sucrose & - & - & $100 \cdot 0$ & $100 \cdot 0$ & $100 \cdot 0$ & - & - & $100 \cdot 0$ & $100 \cdot 0$ & $100 \cdot 0$ & $100 \cdot 0$ \\
\hline Vitamin trace element mix $^{*}$ & $27 \cdot 5$ & $27 \cdot 5$ & 27.5 & 27.5 & 27.5 & 27.5 & 27.5 & 27.5 & 27.5 & 27.5 & 27.5 \\
\hline Mineral mix $\dagger$ & $35 \cdot 0$ & $35 \cdot 0$ & $35 \cdot 0$ & $35 \cdot 0$ & $35 \cdot 0$ & $35 \cdot 0$ & $35 \cdot 0$ & $35 \cdot 0$ & $35 \cdot 0$ & $35 \cdot 0$ & $35 \cdot 0$ \\
\hline Choline chloride & 2.5 & 2.5 & 2.5 & 2.5 & 2.5 & 2.5 & 2.5 & 2.5 & 2.5 & 2.5 & 2.5 \\
\hline Cooked brown rice & 932.0 & - & - & - & - & - & - & - & - & - & - \\
\hline Cooked polished white rice & - & $932 \cdot 0$ & - & - & - & - & - & - & - & - & - \\
\hline Cooked buckwheat & - & - & $664 \cdot 0$ & - & - & - & - & - & - & - & - \\
\hline Cooked oats & - & - & - & 648.0 & - & - & & - & - & - & - \\
\hline Cooked proso millet & - & - & & - & 581.0 & - & - & - & - & - & - \\
\hline Cooked foxtail millet & - & - & - & - & - & 777.0 & - & - & - & - & - \\
\hline Cooked tartary buckwheat & - & - & - & - & & - & $932 \cdot 0$ & - & - & - & - \\
\hline Cooked adlay & - & - & - & - & - & - & - & 519.0 & - & - & - \\
\hline Wheat bread & - & - & - & - & - & - & - & - & 595.0 & - & - \\
\hline Enzyme-hydrolysed casein & - & - & - & - & - & - & - & - & - & - & - \\
\hline Lactic casein & - & - & - & - & - & - & - & - & - & - & $115 \cdot 0$ \\
\hline Titanium dioxide & 3.0 & 3.0 & 3.0 & 3.0 & 3.0 & 3.0 & 3.0 & 3.0 & 3.0 & 3.0 & 3.0 \\
\hline Total $(\mathrm{g})$ & 1000 & 1000 & 1000 & 1000 & 1000 & 1000 & 1000 & 1000 & 1000 & 1000 & 1000 \\
\hline
\end{tabular}

* The vitamins and trace elements are as follows: $250 \mathrm{mg}$ retinol; $1.8 \mathrm{mg}$ cholecalciferol; $1185 \mathrm{mg}$-tocopherol; $1808 \mathrm{mg}$ thiamine; $312 \mathrm{mg}$ riboflavin; $2338 \mathrm{mg}$ niacin; $2058 \mathrm{mg}$ pantothenic acid; $312 \mathrm{mg}$ pyridoxine; $1.8 \mathrm{mg}$ cyanocobalamin; $125 \mathrm{mg}$ phylloquinone; $93.9 \mathrm{mg}$ folic acid; $4.56 \mathrm{~g} \mathrm{Mn} ; 10.29 \mathrm{~g} \mathrm{Fe} ; 904 \mathrm{mg} \mathrm{Cu} ; 3273 \mathrm{mg} \mathrm{Zn;} 41 \mathrm{mg}$ iodine; $7.5 \mathrm{mg} \mathrm{Se} ; 39 \mathrm{mg}$ Co.

† The mineral mix of the diet includes $25 \mathrm{~g} \mathrm{CaPO}_{4}, 5.3 \mathrm{~g} \mathrm{CaCO}_{3}, 3.6 \mathrm{~g} \mathrm{NaCl}, 12.5 \mathrm{~g} \mathrm{KCl}$ and $3.6 \mathrm{~g} \mathrm{MgSO}_{4}$. 
Table 2. Determined crude protein (CP) and amino acid (AA) compositions of cooked brown rice, polished rice, buckwheat, oats, proso millet, foxtail millet, tartary buckwheat, adlay and whole wheat $(\mathrm{g} / \mathrm{kg} \mathrm{DM})^{\star}$

(Mean values with their standard errors)

\begin{tabular}{|c|c|c|c|c|c|c|c|c|c|c|c|c|c|c|c|c|c|c|c|}
\hline & \multicolumn{2}{|c|}{ Brown rice } & \multicolumn{2}{|c|}{ Polished rice } & \multicolumn{2}{|c|}{ Buckwheat } & \multicolumn{2}{|c|}{ Oats } & \multicolumn{2}{|c|}{ Proso millet } & \multicolumn{2}{|c|}{ Foxtail millet } & \multicolumn{2}{|c|}{ Tartary buckwheat } & \multicolumn{2}{|c|}{ Adlay } & \multicolumn{2}{|c|}{ Whole wheat } & \multirow[b]{2}{*}{$P$} \\
\hline & Mean & SEM & Mean & SEM & Mean & SEM & Mean & SEM & Mean & SEM & Mean & SEM & Mean & SEM & Mean & SEM & Mean & SEM & \\
\hline $\mathrm{CP}$ & $96 \cdot 04^{\mathrm{e}}$ & 1.78 & $91.54^{\mathrm{e}}$ & 1.08 & $150.53^{\mathrm{c}}$ & $2 \cdot 1$ & $154.33^{c}$ & 2.85 & $172 \cdot 14^{\mathrm{b}}$ & $4 \cdot 26$ & $128.66^{d}$ & 1.43 & $94.67^{\mathrm{e}}$ & $2 \cdot 23$ & $192 \cdot 81^{\mathrm{a}}$ & 4.35 & $168 \cdot 23^{b}$ & 2.94 & 0.05 \\
\hline \multicolumn{20}{|l|}{ Indispensable AA } \\
\hline His & $2.08^{\mathrm{e}}$ & 0.03 & $2 \cdot 21^{\mathrm{d}, \mathrm{e}}$ & 0.02 & $3.55^{\mathrm{a}}$ & 0.09 & $2.98^{\mathrm{C}}$ & 0.07 & $2.95^{\mathrm{C}}$ & 0.10 & $2 \cdot 28^{\mathrm{d}}$ & 0.07 & $2 \cdot 30^{d}$ & 0.03 & $3 \cdot 24^{\mathrm{b}}$ & 0.10 & $3 \cdot 60^{\mathrm{a}}$ & 0.06 & 0.05 \\
\hline Thr & $3.07^{f}$ & 0.04 & $3.05^{f}$ & 0.07 & $5 \cdot 69^{\mathrm{a}}$ & 0.14 & $4.52^{\mathrm{c}}$ & 0.06 & $4 \cdot 11^{d}$ & 0.05 & $4 \cdot 20^{d}$ & 0.10 & $3.65^{\mathrm{e}}$ & 0.12 & $4.78^{\mathrm{b}}$ & 0.11 & $4.62^{b, c}$ & 0.12 & 0.05 \\
\hline Val & $4.96^{\mathrm{e}}$ & 0.07 & $4.94^{\mathrm{e}}$ & 0.12 & $7.41^{\mathrm{b}}$ & 0.26 & $7 \cdot 15^{\mathrm{b}, \mathrm{c}}$ & 0.13 & $7.04^{c}$ & 0.10 & $5.57^{d}$ & 0.12 & $50.00^{e}$ & 0.05 & $9.54^{\mathrm{a}}$ & 0.14 & $6 \cdot 87^{c}$ & 0.09 & 0.05 \\
\hline Met & $1.66^{f}$ & 0.06 & $2.02^{d}$ & 0.05 & $1.94^{\mathrm{d}, \mathrm{e}}$ & 0.03 & $1.84^{\mathrm{e}}$ & 0.02 & $3.22^{\mathrm{b}}$ & 0.04 & $3.50^{\mathrm{a}}$ & 0.11 & $1.03^{\mathrm{g}}$ & 0.12 & $2 \cdot 20^{\mathrm{c}}$ & 0.07 & $1.64^{\dagger}$ & 0.04 & 0.05 \\
\hline Lys & $2.79^{\mathrm{e}}$ & 0.04 & $2.67^{\mathrm{e}}$ & 0.04 & $8.56^{a}$ & 0.17 & $5.03^{\mathrm{c}}$ & 0.09 & $0.78^{\text {h }}$ & 0.01 & $1 \cdot 18^{\mathrm{g}}$ & 0.04 & $5 \cdot 27^{\mathrm{b}}$ & 0.10 & $2 \cdot 27^{\dagger}$ & 0.07 & $4.44^{d}$ & 0.13 & 0.05 \\
\hline lle & $3.28^{9}$ & 0.09 & $3.49^{9}$ & 0.13 & $5.58^{\mathrm{C}}$ & 0.18 & $5.26^{d}$ & 0.11 & $5.99^{\mathrm{b}}$ & 0.11 & $4.66^{\mathrm{e}}$ & 0.15 & $4.01^{\dagger}$ & 0.11 & $7 \cdot 10^{\mathrm{a}}$ & 0.09 & $5.59^{\mathrm{C}}$ & 0.10 & 0.05 \\
\hline Leu & $7.07^{f}$ & 0.15 & $7.02^{f}$ & 0.07 & $10.06^{e}$ & 0.12 & $10 \cdot 74^{d}$ & 0.24 & $20 \cdot 21^{b}$ & 0.24 & $15.06^{c}$ & 0.23 & $6.90^{f}$ & 0.16 & $26 \cdot 10^{\mathrm{a}}$ & 0.28 & $10.79^{d}$ & 0.16 & 0.05 \\
\hline Phe & $4.39^{\dagger}$ & 0.12 & $4.68^{e, f}$ & 0.10 & $7 \cdot 19^{d}$ & 0.12 & $7 \cdot 65^{c}$ & 0.16 & $9 \cdot 05^{b}$ & 0.10 & $6.92^{d}$ & 0.15 & $4.73^{\mathrm{e}}$ & 0.05 & $9 \cdot 83^{\mathrm{a}}$ & 0.23 & $7.55^{c}$ & 0.15 & 0.05 \\
\hline Trp & $1 \cdot 16^{\mathrm{d}}$ & 0.02 & $0.76^{\dagger}$ & 0.02 & $1.83^{\mathrm{a}}$ & 0.05 & $1.15^{\mathrm{d}}$ & 0.03 & $1.28^{\mathrm{C}}$ & 0.03 & $1.54^{\mathrm{b}}$ & 0.03 & $1.52^{\mathrm{b}}$ & 0.04 & $1.27^{\mathrm{c}}$ & 0.03 & $1.05^{\mathrm{e}}$ & 0.03 & 0.05 \\
\hline \multicolumn{20}{|l|}{ Dispensable AA } \\
\hline Asp & $7 \cdot 86^{\dagger}$ & 0.21 & $7 \cdot 42^{f}$ & 0.12 & $13.88^{a}$ & 0.39 & $11 \cdot 22^{c}$ & 0.19 & $8 \cdot 17^{\mathrm{e}}$ & 0.20 & $7 \cdot 88^{e, f}$ & 0.14 & $9.00^{e}$ & 0.10 & $11.89^{b}$ & 0.30 & $8.12^{\mathrm{e}}$ & 0.15 & 0.05 \\
\hline Ser & $4.50^{\mathrm{g}}$ & 0.06 & $4.43^{\mathrm{g}}$ & 0.11 & $7.47^{c}$ & 0.27 & $7 \cdot 01^{d}$ & 0.09 & $9 \cdot 68^{a}$ & 0.21 & $5.35^{\mathrm{e}}$ & 0.17 & $4.90^{f}$ & 0.10 & $8.51^{b}$ & 0.23 & $7.62^{\mathrm{C}}$ & 0.14 & 0.05 \\
\hline Glu & $15 \cdot 87^{\mathrm{g}}$ & 0.23 & $15 \cdot 31^{\mathrm{g}}$ & 0.21 & $26.50^{\mathrm{e}}$ & 0.83 & $31.05^{d}$ & 0.46 & $33.27^{\mathrm{c}}$ & 0.40 & $23.58^{f}$ & 0.44 & $14.96^{9}$ & 0.17 & $43.93^{b}$ & 0.49 & $46 \cdot 79^{a}$ & 0.90 & 0.05 \\
\hline Gly & $4 \cdot 16^{\mathrm{d}}$ & 0.10 & $3.81^{\mathrm{e}}$ & 0.09 & $8.44^{\mathrm{a}}$ & 0.27 & $6.77^{b}$ & 0.12 & $2.63^{9}$ & 0.06 & $3.04^{f}$ & 0.04 & $5.26^{\mathrm{c}}$ & 0.09 & $4.27^{d}$ & 0.10 & $6.75^{\mathrm{b}}$ & 0.12 & 0.05 \\
\hline Arg & $7 \cdot 51^{d}$ & 0.33 & $7 \cdot 30^{d}$ & 0.12 & $15 \cdot 21^{\mathrm{a}}$ & 0.18 & $9.64^{\mathrm{b}}$ & 0.19 & $4.73^{\mathrm{e}}$ & 0.07 & $4 \cdot 21^{f}$ & 0.12 & $8.69^{c}$ & 0.14 & $8.62^{c}$ & 0.19 & $8.46^{c}$ & 0.19 & 0.05 \\
\hline Ala & $4 \cdot 92^{f}$ & 0.07 & $4.61^{\dagger}$ & 0.12 & $6.09^{\mathrm{d}, \mathrm{e}}$ & 0.07 & $6 \cdot 32^{\mathrm{d}}$ & 0.11 & $15 \cdot 51^{b}$ & 0.39 & $10.06^{c}$ & 0.10 & $4 \cdot 11^{\mathrm{g}}$ & 0.11 & $17 \cdot 62^{\mathrm{a}}$ & 0.24 & $5.73^{\mathrm{e}}$ & 0.06 & 0.05 \\
\hline Pro & $4 \cdot 34^{\mathrm{g}}$ & 0.06 & $4.13^{\mathrm{g}}$ & 0.05 & $6 \cdot 17^{f}$ & 0.09 & $8.06^{\mathrm{e}}$ & 0.15 & $11.25^{\mathrm{c}}$ & 0.14 & $9.24^{d}$ & 0.15 & $3.61^{\mathrm{h}}$ & 0.08 & $15 \cdot 21^{\mathrm{b}}$ & 0.22 & $18.05^{\mathrm{a}}$ & 0.29 & 0.05 \\
\hline Cys & $1.05^{\mathrm{d}, \mathrm{e}}$ & 0.03 & $1 \cdot 14^{\mathrm{d}}$ & 0.04 & $2 \cdot 72^{\mathrm{b}}$ & 0.06 & $2.91^{\mathrm{b}}$ & 0.10 & $0.91^{\mathrm{e}, \mathrm{f}}$ & 0.01 & $3.92^{\mathrm{a}}$ & 0.10 & $0.83^{\dagger}$ & 0.01 & $0.89^{\mathrm{e}, \mathrm{f}}$ & 0.01 & $2 \cdot 18^{\mathrm{C}}$ & 0.22 & 0.05 \\
\hline Tyr & $3.88^{f}$ & 0.12 & $4.31^{\mathrm{e}}$ & 0.13 & $4 \cdot 37^{\mathrm{e}}$ & 0.05 & $5 \cdot 20^{\mathrm{C}}$ & 0.08 & $5 \cdot 88^{\mathrm{b}}$ & 0.09 & $3.82^{f}$ & 0.11 & $2 \cdot 77^{\mathrm{g}}$ & 0.04 & $7 \cdot 62^{\mathrm{a}}$ & 0.21 & $4 \cdot 89^{d}$ & 0.16 & 0.05 \\
\hline Total indispensable AA & $30.47^{f}$ & 0.63 & $30.83^{f}$ & 0.48 & $51.82^{\mathrm{c}}$ & 1.17 & $46 \cdot 33^{d}$ & 0.91 & $54.64^{b}$ & 0.76 & $44.9^{d}$ & 0.99 & $34.40^{\mathrm{e}}$ & 0.78 & $66 \cdot 33^{a}$ & 0.98 & $46 \cdot 14^{d}$ & 0.87 & 0.05 \\
\hline Total dispensable AA & $54 \cdot 10^{f}$ & 1.21 & $52 \cdot 47^{f}$ & 0.98 & $90 \cdot 86^{c, d}$ & $2 \cdot 20$ & $88.19^{d}$ & 1.49 & $92 \cdot 04^{\mathrm{C}}$ & 1.55 & $71.09^{\mathrm{e}}$ & 1.39 & $54 \cdot 14^{f}$ & 0.85 & $118.53^{\mathrm{a}}$ & 2.00 & $108 \cdot 6^{\mathrm{b}}$ & 2.24 & 0.05 \\
\hline Total AA & $84.57^{f}$ & 1.85 & $83.30^{\dagger}$ & 1.47 & $142 \cdot 68^{c}$ & 3.37 & $134.52^{\mathrm{d}}$ & $2 \cdot 40$ & $146.68^{c}$ & $2 \cdot 31$ & $115.99^{\mathrm{e}}$ & $2 \cdot 38$ & $88.54^{\dagger}$ & 1.62 & $184.86^{a}$ & 2.98 & $154.74^{\mathrm{b}}$ & 3.11 & 0.05 \\
\hline
\end{tabular}

a,b,c,d,e,f,g,h Mean values within a row with unlike superscript letters were significantly different $(P<0.05)$.

* Based on triplicate determinations. CP was based on a N-to-protein conversion factor of 6.25. 
Table 3. Determined amino acid (AA) compositions of brown rice, polished rice, buckwheat, oats, proso millet, foxtail millet, tartary buckwheat, adlay and whole wheat-based diets (g/kg DM) ${ }^{\star}$ (Mean values with their standard errors)

\begin{tabular}{|c|c|c|c|c|c|c|c|c|c|c|c|c|c|c|c|c|c|c|c|}
\hline & \multicolumn{2}{|c|}{ Brown rice } & \multicolumn{2}{|c|}{ Polished rice } & \multicolumn{2}{|c|}{ Buckwheat } & \multicolumn{2}{|c|}{ Oats } & \multicolumn{2}{|c|}{ Proso millet } & \multicolumn{2}{|c|}{ Foxtail millet } & \multicolumn{2}{|c|}{ Tartary buckwheat } & \multicolumn{2}{|c|}{ Adlay } & \multicolumn{2}{|c|}{ Whole wheat } & \multirow[b]{2}{*}{$P$} \\
\hline & Mean & SEM & Mean & SEM & Mean & SEM & Mean & SEM & Mean & SEM & Mean & SEM & Mean & SEM & Mean & SEM & Mean & SEM & \\
\hline \multicolumn{20}{|l|}{ Indispensable AA } \\
\hline $\mathrm{His}$ & $1.89^{\mathrm{e}}$ & 0.03 & $1.60^{f}$ & 0.03 & $2 \cdot 14^{\mathrm{b}}$ & 0.04 & $2 \cdot 01^{\mathrm{c}, \mathrm{d}}$ & 0.03 & $2.08^{b, c}$ & 0.05 & $1.89^{\mathrm{e}}$ & 0.06 & $2 \cdot 04^{\mathrm{b}, \mathrm{c}}$ & 0.03 & $1.92^{\mathrm{d}, \mathrm{e}}$ & 0.06 & $2 \cdot 28^{\mathrm{a}}$ & 0.05 & 0.05 \\
\hline Thr & $2 \cdot 78^{\mathrm{e}}$ & 0.03 & $2 \cdot 46^{f}$ & 0.03 & $3.46^{\mathrm{a}}$ & 0.06 & $3 \cdot 13^{c}$ & 0.04 & $2 \cdot 99^{d}$ & 0.07 & $3.33^{\mathrm{b}}$ & 0.05 & $3 \cdot 23^{b, c}$ & 0.06 & $2 \cdot 75^{\mathrm{e}}$ & 0.03 & $2 \cdot 95^{\mathrm{d}}$ & 0.09 & 0.05 \\
\hline Val & $4 \cdot 61^{\mathrm{b}}$ & 0.07 & $4 \cdot 18^{\mathrm{C}}$ & 0.05 & $4.61^{\mathrm{b}}$ & 0.15 & $5 \cdot 17^{\mathrm{a}}$ & 0.05 & $5 \cdot 26^{\mathrm{a}}$ & 0.20 & $4 \cdot 15^{\mathrm{C}}$ & 0.09 & $4.54^{b}$ & 0.07 & $5 \cdot 40^{\mathrm{a}}$ & 0.14 & $4.51^{\mathrm{b}}$ & 0.06 & 0.05 \\
\hline Met & $0.96^{\mathrm{d}}$ & 0.03 & $1.62^{\mathrm{C}}$ & 0.03 & $1 \cdot 17^{d}$ & 0.02 & $0.64^{\mathrm{e}}$ & 0.07 & $2 \cdot 15^{\mathrm{b}}$ & 0.35 & $3.05^{\mathrm{a}}$ & 0.05 & $1.02^{\mathrm{d}}$ & 0.01 & $2.07^{\mathrm{b}}$ & 0.04 & $1 \cdot 10^{\mathrm{d}}$ & 0.02 & 0.05 \\
\hline Lys & $2 \cdot 31^{d}$ & 0.03 & $1.97^{\mathrm{e}}$ & 0.03 & $4.89^{\mathrm{a}}$ & 0.18 & $3.07^{\mathrm{c}}$ & 0.08 & $0.36^{i}$ & 0.00 & $0.70^{\mathrm{h}}$ & 0.01 & $4 \cdot 40^{\mathrm{b}}$ & 0.08 & $0.92^{9}$ & 0.02 & $1.32^{f}$ & 0.01 & 0.05 \\
\hline Ile & $3.09^{\mathrm{e}}$ & 0.06 & $2 \cdot 85^{f}$ & 0.04 & $3.39^{d}$ & 0.12 & $3 \cdot 71^{\mathrm{c}}$ & 0.07 & $4.49^{\mathrm{a}}$ & 0.09 & $4 \cdot 08^{b}$ & 0.05 & $3.58^{\mathrm{c}}$ & 0.04 & $4 \cdot 01^{b}$ & 0.11 & $3.66^{\mathrm{c}}$ & 0.10 & 0.05 \\
\hline Leu & $6 \cdot 83^{e}$ & $0 \cdot 10$ & $6 \cdot 24^{f}$ & 0.11 & $6 \cdot 48^{e, f}$ & 0.11 & $7.94^{\mathrm{c}}$ & 0.14 & $15 \cdot 37^{a}$ & 0.46 & $13 \cdot 20^{b}$ & 0.21 & $6 \cdot 24^{\dagger}$ & 0.11 & $15 \cdot 44^{\mathrm{a}}$ & 0.32 & $7.39^{d}$ & 0.10 & 0.05 \\
\hline Phe & $4.54^{\mathrm{d}, \mathrm{e}}$ & 0.06 & $3.93^{f}$ & 0.05 & $4.59^{\mathrm{d}}$ & 0.15 & $5.53^{\mathrm{b}}$ & 0.08 & $6 \cdot 76^{a}$ & 0.19 & $5 \cdot 72^{b}$ & 0.09 & $4.31^{\mathrm{e}}$ & 0.10 & $5.65^{b}$ & 0.15 & $4.98^{\mathrm{C}}$ & 0.09 & 0.05 \\
\hline Trp & $1.94^{\mathrm{a}}$ & 0.07 & $0.75^{f}$ & 0.02 & $1 \cdot 11^{\mathrm{d}}$ & 0.03 & $1.36^{\mathrm{c}}$ & 0.03 & $0.94^{\mathrm{e}}$ & 0.01 & $1.78^{\mathrm{b}}$ & 0.03 & $1.37^{\mathrm{C}}$ & 0.03 & $0.95^{\mathrm{e}}$ & 0.02 & $0.99^{e}$ & 0.02 & 0.05 \\
\hline \multicolumn{20}{|l|}{ Dispensable AA } \\
\hline Asp & $7.07^{\mathrm{c}}$ & 0.09 & $6.52^{d}$ & 0.16 & $8.87^{\mathrm{a}}$ & 0.29 & $7.92^{\mathrm{a}}$ & 0.09 & $6.05^{\mathrm{e}}$ & 0.13 & $6.46^{\mathrm{d}}$ & 0.08 & $8.08^{\mathrm{a}}$ & 0.10 & $6.68^{d}$ & 0.17 & $5 \cdot 23^{f}$ & 0.08 & 0.05 \\
\hline Ser & $4 \cdot 14^{d}$ & 0.06 & $3.85^{\mathrm{e}}$ & 0.04 & $4.76^{\mathrm{c}}$ & 0.12 & $5 \cdot 16^{\mathrm{b}}$ & 0.06 & $7 \cdot 25^{a}$ & 0.18 & $4.29^{d}$ & 0.07 & $4 \cdot 36^{\mathrm{d}}$ & 0.05 & $4.82^{\mathrm{C}}$ & 0.16 & $4.94^{b, c}$ & 0.11 & 0.05 \\
\hline Glu & $14.52^{f}$ & 0.25 & $14.00^{f}$ & 0.22 & $17 \cdot 04^{e}$ & 0.60 & $22 \cdot 36^{c}$ & 0.32 & $24.90^{\mathrm{b}}$ & 0.79 & $19.51^{d}$ & 0.21 & $13 \cdot 52^{f}$ & 0.19 & $24.59^{b}$ & 0.51 & $30.75^{\mathrm{a}}$ & 0.36 & 0.05 \\
\hline Gly & $3.79^{e}$ & 0.05 & $3.30^{f}$ & 0.08 & $5 \cdot 43^{\mathrm{a}}$ & 0.14 & $5 \cdot 16^{b}$ & 0.11 & $1.86^{\mathrm{h}}$ & 0.04 & $2.39^{9}$ & 0.07 & $4.69^{\mathrm{C}}$ & 0.05 & $2 \cdot 44^{\mathrm{g}}$ & 0.06 & $4.43^{\mathrm{d}}$ & 0.08 & 0.05 \\
\hline Arg & $7 \cdot 27^{\mathrm{c}}$ & 0.19 & $6.04^{d}$ & 0.08 & $9.81^{a}$ & 0.14 & $7 \cdot 16^{c}$ & 0.14 & $1.48^{\mathrm{h}}$ & 0.03 & $2.39^{9}$ & 0.07 & $7.63^{\mathrm{b}}$ & 0.13 & $4 \cdot 22^{\dagger}$ & 0.13 & $5.29^{e}$ & 0.11 & 0.05 \\
\hline Ala & $4.71^{d}$ & 0.05 & $4.38^{\mathrm{e}}$ & 0.04 & $3.88^{f}$ & 0.14 & $4.72^{\mathrm{d}}$ & 0.06 & $12 \cdot 19^{\mathrm{a}}$ & 0.20 & $8.41^{\mathrm{c}}$ & $0 \cdot 18$ & $3.83^{f}$ & 0.10 & $10 \cdot 31^{b}$ & 0.17 & $3 \cdot 71^{f}$ & $0 \cdot 10$ & 0.05 \\
\hline Pro & $4 \cdot 12^{\mathrm{e}}$ & 0.06 & $3.51^{f}$ & 0.05 & $3.68^{f}$ & 0.05 & $5 \cdot 83^{d}$ & 0.08 & $8 \cdot 63^{b}$ & 0.25 & $7.90^{\mathrm{c}}$ & 0.15 & $3 \cdot 20^{9}$ & 0.03 & $8.91^{b}$ & 0.13 & $11.54^{\mathrm{a}}$ & 0.18 & 0.05 \\
\hline Cys & $0.62^{\mathrm{c}}$ & 0.01 & $0.62^{\mathrm{c}}$ & 0.02 & $1 \cdot 12^{\mathrm{b}}$ & 0.02 & $1.42^{\mathrm{a}}$ & 0.25 & $0.60^{c}$ & 0.01 & $0.69^{c}$ & 0.01 & $0.61^{\mathrm{C}}$ & 0.02 & $0.59^{\mathrm{c}}$ & 0.01 & $1.06^{b}$ & 0.02 & 0.05 \\
\hline Tyr & $3.57^{b, c}$ & 0.06 & $3.37^{d}$ & 0.05 & $2 \cdot 58^{\mathrm{e}}$ & 0.04 & $3 \cdot 62^{b}$ & 0.11 & $4 \cdot 36^{\mathrm{a}}$ & 0.12 & $3 \cdot 39^{c, d}$ & 0.09 & $2.43^{\mathrm{e}}$ & 0.09 & $4 \cdot 36^{\mathrm{a}}$ & 0.09 & $3 \cdot 24^{d}$ & 0.04 & 0.05 \\
\hline Total indispensable AA & $28.95^{\mathrm{e}}$ & 0.08 & $25 \cdot 61^{f}$ & 0.39 & $31 \cdot 84^{\mathrm{c}, \mathrm{d}}$ & 0.66 & $32 \cdot 58^{\mathrm{c}}$ & 0.60 & $40 \cdot 41^{a}$ & 1.40 & $37.91^{\mathrm{b}}$ & 0.65 & $30 \cdot 72^{d}$ & 0.52 & $39.11^{a, b}$ & 0.58 & $29 \cdot 17^{e}$ & 0.35 & 0.05 \\
\hline Total dispensable AA & $49.80^{e}$ & 0.09 & $45 \cdot 60^{f}$ & 0.34 & $57 \cdot 18^{\mathrm{d}}$ & 1.32 & $63 \cdot 36^{c}$ & 1.04 & $67.32^{\mathrm{b}}$ & 1.75 & $56 \cdot 35^{d}$ & 0.89 & $48 \cdot 36^{\mathrm{e}}$ & 0.77 & $66 \cdot 92^{\mathrm{b}}$ & 1.41 & $70 \cdot 19^{a}$ & 1.08 & 0.05 \\
\hline Total AA & $78 \cdot 75^{e}$ & 0.01 & $71 \cdot 21^{f}$ & 0.73 & $89.02^{d}$ & 1.98 & $95 \cdot 93^{\mathrm{b}, \mathrm{c}}$ & 1.63 & $107 \cdot 74^{\mathrm{a}}$ & 3.14 & $94 \cdot 25^{\mathrm{c}}$ & 1.53 & $79.08^{\mathrm{e}}$ & 1.29 & $106 \cdot 03^{\mathrm{a}}$ & 1.98 & $99 \cdot 36^{\mathrm{b}}$ & 1.43 & 0.05 \\
\hline
\end{tabular}

a,b,c,d,e,f,g,h Mean values within a row with unlike superscript letters were significantly different $(P<0.05)$.

* Based on triplicate determinations. Crude protein was based on a N-to-protein conversion factor of 6.25 . 
Table 4. Mean apparent ileal digestibility of amino acid (AA) in brown rice, polished rice, buckwheat, oats, proso millet, foxtail millet, tartary buckwheat, adlay and whole wheat (\%) (Mean values with their standard errors)

\begin{tabular}{|c|c|c|c|c|c|c|c|c|c|c|c|c|c|c|c|c|c|c|c|c|}
\hline & \multicolumn{2}{|c|}{ Brown rice } & \multicolumn{2}{|c|}{ Polished rice } & \multicolumn{2}{|c|}{ Buckwheat } & \multicolumn{2}{|c|}{ Oats } & \multicolumn{2}{|c|}{ Proso millet } & \multicolumn{2}{|c|}{ Foxtail millet } & \multicolumn{2}{|c|}{ Tartary buckwheat } & \multicolumn{2}{|c|}{ Adlay } & \multicolumn{2}{|c|}{ Whole wheat } & \multirow[b]{2}{*}{ SEM } & \multirow[b]{2}{*}{$P$} \\
\hline & Mean & SEM & Mean & SEM & Mean & SEM & Mean & SEM & Mean & SEM & Mean & SEM & Mean & SEM & Mean & SEM & Mean & SEM & & \\
\hline \multicolumn{21}{|c|}{ Indispensable AA } \\
\hline His & $80.93^{a, b}$ & $2 \cdot 76$ & $68 \cdot 11^{d}$ & 4.82 & $78.88^{\mathrm{b}, \mathrm{c}}$ & 3.37 & $78.03^{\mathrm{b}, \mathrm{c}}$ & 4.44 & $74 \cdot 34^{\mathrm{c}}$ & $4 \cdot 18$ & $81 \cdot 69^{a, b}$ & 4.03 & $67 \cdot 18^{d}$ & 3.95 & $81.98^{a, b}$ & 3.67 & $85.46^{a}$ & 3.65 & $2 \cdot 11$ & 0.05 \\
\hline Thr & $64 \cdot 51^{\mathrm{b}, \mathrm{c}}$ & 3.32 & $42 \cdot 67^{f}$ & 3.31 & $67 \cdot 01^{a, b}$ & 2.09 & $60 \cdot 81^{\mathrm{c}}$ & 3.08 & $49.63^{\mathrm{e}}$ & 2.74 & $65 \cdot 10^{\mathrm{b}, \mathrm{c}}$ & 2.45 & $55 \cdot 11^{d}$ & 2.73 & $69.54^{\mathrm{a}, \mathrm{b}}$ & 3.36 & $70 \cdot 70^{\mathrm{a}}$ & 5.59 & 3.19 & 0.05 \\
\hline Val & $77 \cdot 15^{\mathrm{c}}$ & 1.43 & $72 \cdot 36^{d}$ & 1.84 & $78.48^{\mathrm{b}, \mathrm{c}}$ & $1 \cdot 70$ & $79.88^{b, c}$ & 1.72 & $73 \cdot 21^{d}$ & 2.30 & $81.02^{\mathrm{b}}$ & 1.88 & $70 \cdot 74^{d}$ & $2 \cdot 14$ & $87.43^{\mathrm{a}}$ & 1.58 & $85.59^{a}$ & 1.68 & 1.92 & 0.05 \\
\hline Met & $56 \cdot 60^{\mathrm{d}}$ & 0.90 & $55.44^{\mathrm{d}}$ & 4.82 & $84.91^{\mathrm{a}, \mathrm{b}}$ & 1.17 & $82 \cdot 12^{\mathrm{b}}$ & 1.28 & $65.96^{c}$ & 1.42 & $86 \cdot 90^{\mathrm{a}, \mathrm{b}}$ & 4.57 & $64 \cdot 77^{c}$ & 7.62 & $89 \cdot 19^{a}$ & 3.91 & $88.69^{\mathrm{a}}$ & $2 \cdot 16$ & 4.69 & 0.05 \\
\hline Lys & $77 \cdot 63^{\mathrm{a}, \mathrm{b}}$ & 3.61 & $73.93^{\mathrm{a}, \mathrm{b}, \mathrm{c}}$ & 3.81 & $79.70^{\mathrm{a}}$ & 1.63 & $71 \cdot 39^{\mathrm{a}, \mathrm{b}, \mathrm{c}}$ & 2.65 & $17 \cdot 25^{f}$ & $2 \cdot 37$ & $36 \cdot 18^{e}$ & 11.65 & $65 \cdot 55^{\mathrm{c}}$ & 1.94 & $50.97^{d}$ & 3.58 & $68.56^{\mathrm{b}, \mathrm{c}}$ & $2 \cdot 61$ & 7.07 & 0.05 \\
\hline Ile & $73.57^{d}$ & 3.89 & $68.79^{e}$ & 2.71 & $79.52^{c}$ & 2.47 & $83.71^{\mathrm{b}}$ & $2 \cdot 11$ & $72 \cdot 15^{\mathrm{d}, \mathrm{e}}$ & 1.84 & $79 \cdot 98^{c}$ & 1.95 & $71 \cdot 06^{\mathrm{d}, \mathrm{e}}$ & 2.67 & $87 \cdot 75^{a}$ & $2 \cdot 19$ & $87.86^{\mathrm{a}}$ & $2 \cdot 24$ & 2.41 & 0.05 \\
\hline Leu & $75.97^{f}$ & 1.59 & $68 \cdot 16^{\mathrm{h}}$ & 1.32 & $81 \cdot 11^{d}$ & 1.00 & $86 \cdot 10^{C}$ & 1.03 & $78 \cdot 83^{\mathrm{e}}$ & 1.38 & $89 \cdot 68^{\mathrm{b}}$ & $1 \cdot 18$ & $71 \cdot 84^{9}$ & 1.50 & $93 \cdot 84^{a}$ & 1.04 & $89.08^{\mathrm{b}}$ & 2.22 & 2.90 & 0.05 \\
\hline Phe & $77 \cdot 42^{\mathrm{c}}$ & $2 \cdot 14$ & $74.57^{d}$ & 1.89 & $84.87^{\mathrm{b}}$ & 1.75 & $85 \cdot 37^{\mathrm{b}}$ & 1.55 & $76 \cdot 27^{\mathrm{c}, \mathrm{d}}$ & 1.23 & $86 \cdot 33^{\mathrm{b}}$ & 2.62 & $76 \cdot 47^{\mathrm{c}, \mathrm{d}}$ & 2.06 & $91 \cdot 77^{\mathrm{a}}$ & 1.14 & $90 \cdot 97^{\mathrm{a}}$ & 1.28 & 2.20 & 0.05 \\
\hline Trp & $83.05^{b, c}$ & 1.43 & $71.99^{\mathrm{e}, \mathrm{f}}$ & 0.91 & $79.79^{c, d}$ & 1.21 & $74.54^{\mathrm{e}}$ & 1.31 & $70 \cdot 39^{f}$ & 3.13 & $86 \cdot 87^{\mathrm{a}}$ & 1.69 & $78 \cdot 37^{d}$ & 2.33 & $83.93^{a, b}$ & 2.90 & $84 \cdot 22^{a, b}$ & 4.03 & 1.95 & 0.05 \\
\hline Mean & $74.09^{d}$ & 1.41 & $66 \cdot 22^{e, f}$ & 2.43 & $79.36^{b, c}$ & 1.76 & $78 \cdot 00^{\mathrm{C}}$ & 1.80 & $64 \cdot 23^{f}$ & 2.03 & $77.08^{\mathrm{c}, \mathrm{d}}$ & $3 \cdot 12$ & $69.01^{\mathrm{e}}$ & 2.78 & $81 \cdot 82^{a, b}$ & $2 \cdot 24$ & $83.46^{\mathrm{a}}$ & 2.44 & 2.30 & 0.05 \\
\hline \multicolumn{21}{|c|}{ Dispensable AA } \\
\hline Asp & $75 \cdot 98^{\mathrm{b}}$ & 1.69 & $64.03^{d}$ & 2.63 & $80 \cdot 30^{a}$ & 1.33 & $76 \cdot 89^{b}$ & 1.21 & $59.49^{e}$ & 1.47 & $75 \cdot 97^{b}$ & $2 \cdot 34$ & $65.92^{d}$ & 1.35 & $77 \cdot 65^{\mathrm{b}}$ & 1.27 & $70 \cdot 71^{\mathrm{c}}$ & 1.64 & $2 \cdot 40$ & 0.05 \\
\hline Ser & $70.59^{\mathrm{c}, \mathrm{c}}$ & $2 \cdot 24$ & $54.48^{\mathrm{e}}$ & 3.79 & $68 \cdot 76^{c}$ & 1.25 & $70.07^{\mathrm{c}}$ & 2.05 & $63 \cdot 01^{d}$ & 1.32 & $75 \cdot 38^{\mathrm{b}}$ & 2.07 & $62 \cdot 27^{d}$ & $2 \cdot 22$ & $80 \cdot 38^{a}$ & 2.41 & $81 \cdot 24^{a}$ & 1.87 & 2.92 & 0.05 \\
\hline Glu & $74 \cdot 39^{f}$ & 1.17 & $63.88^{h}$ & 1.42 & $86 \cdot 50^{d}$ & 0.82 & $89.54^{\mathrm{C}}$ & 0.67 & $66 \cdot 33^{9}$ & 1.09 & $84.62^{\mathrm{e}}$ & 0.96 & $75.09^{h}$ & 0.74 & $92 \cdot 06^{\mathrm{b}}$ & 0.81 & $94 \cdot 12^{\mathrm{a}}$ & 0.47 & 3.72 & 0.05 \\
\hline Gly & $64 \cdot 44^{\mathrm{b}}$ & 1.45 & $41.35^{\mathrm{d}}$ & 2.73 & $67.46^{\mathrm{a}, \mathrm{b}}$ & 2.09 & $65.49^{\mathrm{b}}$ & 1.56 & $9.86^{f}$ & 4.59 & $31.28^{\mathrm{e}}$ & 3.32 & $52 \cdot 10^{c}$ & 1.97 & $41.57^{d}$ & 3.55 & $69 \cdot 74^{\mathrm{a}}$ & 1.97 & 6.72 & 0.05 \\
\hline Arg & $87.54^{\mathrm{a}, \mathrm{b}}$ & 1.26 & $80 \cdot 20^{\mathrm{d}, \mathrm{e}}$ & 1.67 & $87.74^{\mathrm{a}}$ & 1.47 & $83.46^{\mathrm{b}, \mathrm{c}, \mathrm{d}, \mathrm{e}}$ & $1 \cdot 10$ & $58.75^{f}$ & 5.63 & $79 \cdot 37^{e}$ & 2.44 & $82 \cdot 58^{\mathrm{c}, \mathrm{d}, \mathrm{e}}$ & 1.65 & $86 \cdot 56^{\mathrm{a}, \mathrm{b}, \mathrm{c}}$ & 3.30 & $84 \cdot 07^{\mathrm{a}, \mathrm{b}, \mathrm{c}, \mathrm{d}}$ & 1.69 & 2.97 & 0.05 \\
\hline Ala & $77 \cdot 25^{\mathrm{b}}$ & 0.90 & $67.82^{\mathrm{d}}$ & 2.80 & $72 \cdot 28^{c}$ & 1.62 & $77.35^{\mathrm{b}}$ & 1.74 & $74 \cdot 17^{\mathrm{C}}$ & 1.37 & $85.99^{a}$ & 1.29 & $66.82^{d}$ & 1.99 & $86.93^{a}$ & 0.92 & $79.49^{b}$ & 2.39 & 2.36 & 0.05 \\
\hline Pro & $70.95^{c}$ & $2 \cdot 22$ & $59 \cdot 30^{\mathrm{e}}$ & 3.60 & $66 \cdot 83^{d}$ & 2.49 & $80.07^{b}$ & 1.52 & $69.82^{\mathrm{c}, \mathrm{d}}$ & 1.85 & $85 \cdot 17^{\mathrm{a}}$ & 1.66 & $51.45^{f}$ & 3.32 & $82 \cdot 88^{a, b}$ & 0.86 & $80 \cdot 90^{\mathrm{b}}$ & 1.07 & 3.82 & 0.05 \\
\hline Cys & $55 \cdot 16^{\mathrm{d}, \mathrm{e}}$ & 1.51 & $48 \cdot 40^{\dagger}$ & 1.49 & $75 \cdot 24^{\mathrm{b}}$ & 1.07 & $87.33^{\mathrm{a}}$ & 0.93 & $52 \cdot 07^{\mathrm{e}, \mathrm{f}}$ & 3.07 & $76.50^{\mathrm{b}}$ & 4.05 & $59 \cdot 14^{d}$ & 4.00 & $70 \cdot 18^{\mathrm{c}}$ & $5 \cdot 30$ & $78.00^{\mathrm{b}}$ & 2.86 & 4.52 & 0.05 \\
\hline Tyr & $71.47^{d}$ & 2.49 & $67.74^{\mathrm{d}, \mathrm{e}}$ & 2.45 & $80 \cdot 28^{\mathrm{b}, \mathrm{c}}$ & 3.12 & $83 \cdot 50^{\mathrm{a}, \mathrm{b}}$ & $2 \cdot 22$ & $77 \cdot 25^{c}$ & 2.45 & $83 \cdot 52^{\mathrm{a}, \mathrm{b}}$ & 2.92 & $64.08^{\mathrm{e}}$ & 3.46 & $83 \cdot 77^{\mathrm{a}, \mathrm{b}}$ & 1.82 & $85.07^{a}$ & 2.69 & $2 \cdot 61$ & 0.05 \\
\hline Mean & $71.97^{d}$ & 0.36 & $60 \cdot 80^{f}$ & 1.93 & $76 \cdot 16^{\mathrm{b}, \mathrm{c}}$ & 1.52 & $79 \cdot 30^{\mathrm{a}}$ & $1 \cdot 21$ & $58.97^{\dagger}$ & 1.98 & $75 \cdot 31^{\mathrm{C}}$ & 2.03 & $64.39^{e}$ & 1.90 & $78 \cdot 00^{a, b}$ & 1.83 & $80 \cdot 37^{a}$ & 1.58 & 2.74 & 0.05 \\
\hline Total mean & $73.03^{d}$ & 0.75 & $63.51^{f}$ & $2 \cdot 18$ & $77.76^{\mathrm{b}, \mathrm{c}}$ & 1.57 & $78.65^{\mathrm{b}, \mathrm{c}}$ & 1.49 & $61.60^{f}$ & 1.94 & $76 \cdot 20^{c}$ & 2.57 & $66 \cdot 70^{\mathrm{e}}$ & 2.32 & $79.91^{a, b}$ & 1.98 & $81.92^{\mathrm{a}}$ & 2.01 & 2.50 & 0.05 \\
\hline
\end{tabular}

a,b,c,d,e,f Mean values within a row with unlike superscript letters were significantly different $(P<0.05)$. 
Table 5. Mean true ileal digestibility (TID) of amino acid (AA) in brown rice, polished rice, buckwheat, oats, proso millet, foxtail millet, tartary buckwheat, adlay and whole wheat (\%)* (Mean values with their standard errors)

\begin{tabular}{|c|c|c|c|c|c|c|c|c|c|c|c|c|c|c|c|c|c|c|c|c|}
\hline & \multicolumn{2}{|c|}{ Brown rice } & \multicolumn{2}{|c|}{ Polished rice } & \multicolumn{2}{|c|}{ Buckwheat } & \multicolumn{2}{|c|}{ Oats } & \multicolumn{2}{|c|}{ Proso millet } & \multicolumn{2}{|c|}{ Foxtail millet } & \multicolumn{2}{|c|}{ Tartary buckwheat } & \multicolumn{2}{|c|}{ Adlay } & \multicolumn{2}{|c|}{ Whole wheat } & \multirow[b]{2}{*}{ SEM } & \multirow[b]{2}{*}{$P$} \\
\hline & Mean & SEM & Mean & SEM & Mean & SEM & Mean & SEM & Mean & SEM & Mean & SEM & Mean & SEM & Mean & SEM & Mean & SEM & & \\
\hline \multicolumn{21}{|c|}{ Indispensable AA } \\
\hline His & $93 \cdot 12^{\mathrm{a}}$ & $2 \cdot 76$ & $82.46^{\mathrm{d}, \mathrm{e}}$ & 4.82 & $89.66^{a, b}$ & 3.37 & $89 \cdot 48^{\mathrm{a}, \mathrm{b}}$ & 4.44 & $85 \cdot 42^{c, d}$ & $4 \cdot 18$ & $93 \cdot 88^{\mathrm{a}}$ & 4.03 & $78.46^{e}$ & 3.95 & $94.00^{a}$ & 3.67 & $95.56^{\mathrm{a}}$ & 3.65 & 1.96 & 0.05 \\
\hline Thr & $88.09^{b}$ & 3.32 & $69.24^{e}$ & $3 \cdot 31$ & $85.95^{b, c}$ & 2.09 & $81.76^{\mathrm{C}}$ & 3.08 & $71.56^{\mathrm{e}}$ & 2.74 & $84.76^{b, c}$ & $2 \cdot 45$ & $75.41^{d}$ & 2.73 & $93.39^{a}$ & 3.36 & $92 \cdot 92^{\mathrm{a}}$ & 5.59 & 2.94 & 0.05 \\
\hline Val & $85.91^{c}$ & 1.43 & $82.02^{c}$ & 1.84 & $87 \cdot 25^{\mathrm{a}}$ & 1.70 & $87 \cdot 69^{a}$ & 1.72 & $80 \cdot 89^{b}$ & $2 \cdot 30$ & $90.75^{\mathrm{a}}$ & 1.88 & $79 \cdot 65^{b}$ & $2 \cdot 14$ & $94.91^{a}$ & 1.58 & $94.55^{\mathrm{a}}$ & 1.68 & 1.87 & 0.05 \\
\hline Met & $62.56^{\mathrm{c}}$ & 0.90 & $58.69^{c}$ & 4.82 & $89.39^{a}$ & 1.17 & $90 \cdot 32^{\mathrm{a}}$ & 1.28 & $68 \cdot 41^{\mathrm{b}}$ & 1.42 & $88.63^{\mathrm{a}}$ & 4.57 & $69.93^{b}$ & 7.62 & $91 \cdot 74^{\mathrm{a}}$ & 3.91 & $93.49^{\mathrm{a}}$ & $2 \cdot 16$ & 4.68 & 0.05 \\
\hline Lys & $93 \cdot 41^{a, b}$ & 3.61 & $92 \cdot 48^{\mathrm{a}, \mathrm{b}}$ & 3.81 & $87 \cdot 15^{\mathrm{b}, \mathrm{c}}$ & 1.63 & $83.25^{\mathrm{C}}$ & 2.65 & $95 \cdot 97^{\mathrm{a}}$ & 5.37 & $88 \cdot 27^{\mathrm{b}, \mathrm{c}}$ & 7.65 & $73.84^{d}$ & 1.94 & $90 \cdot 40^{\mathrm{a}, \mathrm{b}, \mathrm{c}}$ & 3.58 & $96 \cdot 18^{\mathrm{a}}$ & $2 \cdot 61$ & $2 \cdot 36$ & 0.05 \\
\hline Ile & $82.87^{\mathrm{e}}$ & 3.89 & $78.87^{f}$ & $2 \cdot 71$ & $88 \cdot 00^{c, d}$ & 2.47 & $91.45^{\mathrm{b}, \mathrm{c}}$ & $2 \cdot 11$ & $78.54^{f}$ & 1.84 & $87.03^{d}$ & 1.95 & $79.07^{f}$ & $2 \cdot 67$ & $94 \cdot 91^{\mathrm{a}, \mathrm{b}}$ & $2 \cdot 19$ & $95 \cdot 70^{\mathrm{a}}$ & $2 \cdot 24$ & $2 \cdot 27$ & 0.05 \\
\hline Leu & $83.49^{d}$ & 1.59 & $76 \cdot 39^{d}$ & 1.32 & $89.03^{\mathrm{c}}$ & 1.00 & $92 \cdot 57^{\mathrm{b}}$ & 1.03 & $82 \cdot 17^{\mathrm{d}}$ & 1.38 & $93.56^{b}$ & $1 \cdot 18$ & $80.07^{\mathrm{e}}$ & 1.50 & $97 \cdot 16^{\mathrm{a}}$ & 1.04 & $96.03^{\mathrm{a}}$ & $2 \cdot 22$ & 2.51 & 0.05 \\
\hline Phe & $83.63^{\mathrm{C}}$ & $2 \cdot 14$ & $81 \cdot 40^{d}$ & 1.89 & $90 \cdot 72^{b}$ & 1.75 & $90 \cdot 22^{b}$ & 1.55 & $80 \cdot 23^{c, d}$ & 1.23 & $91.01^{b}$ & 2.62 & $82.69^{\mathrm{c}, \mathrm{d}}$ & 2.06 & $96 \cdot 51^{a}$ & 1.14 & $96 \cdot 36^{a}$ & 1.28 & 2.09 & 0.05 \\
\hline Trp & $86 \cdot 89^{b}$ & 1.43 & $81.86^{\mathrm{c}, \mathrm{d}}$ & 0.91 & $86 \cdot 50^{b}$ & 1.21 & $80 \cdot 01^{\mathrm{d}, \mathrm{e}}$ & 1.31 & $78.31^{e}$ & 3.13 & $91.05^{\mathrm{a}}$ & 1.69 & $83 \cdot 82^{\mathrm{b}, \mathrm{c}}$ & 2.33 & $91 \cdot 74^{\mathrm{a}}$ & 2.90 & $91.75^{\mathrm{a}}$ & 4.03 & 1.70 & 0.05 \\
\hline Mean & $84.44^{c}$ & 1.41 & $78 \cdot 16^{\mathrm{d}}$ & 2.43 & $88 \cdot 18^{b}$ & 1.76 & $87.42^{\mathrm{b}, \mathrm{c}}$ & 1.80 & $80 \cdot 17^{d}$ & 2.03 & $89 \cdot 88^{b}$ & $3 \cdot 12$ & $78 \cdot 11^{d}$ & $2 \cdot 78$ & $93 \cdot 86^{a}$ & $2 \cdot 24$ & $94.73^{\mathrm{a}}$ & 2.44 & $2 \cdot 11$ & 0.05 \\
\hline \multicolumn{21}{|c|}{ Dispensable AA } \\
\hline Asp & $89.87^{a, b}$ & 1.69 & $79.03^{c}$ & 2.63 & $91 \cdot 36^{a, b}$ & 1.33 & $89 \cdot 28^{b}$ & 1.21 & $75 \cdot 72^{d}$ & 1.47 & $91 \cdot 17^{\mathrm{a}, \mathrm{b}}$ & $2 \cdot 34$ & $78.06^{\mathrm{c}, \mathrm{d}}$ & 1.35 & $92 \cdot 33^{\mathrm{a}}$ & 1.27 & $89.64^{a, b}$ & 1.64 & $2 \cdot 21$ & 0.05 \\
\hline Ser & $88.84^{\mathrm{C}}$ & $2 \cdot 24$ & $74.04^{f}$ & 3.79 & $84.63^{\mathrm{d}}$ & 1.25 & $84.69^{d}$ & 2.05 & $73.42^{f}$ & 1.32 & $93.00^{\mathrm{b}}$ & 2.07 & $79.58^{\mathrm{e}}$ & $2 \cdot 22$ & $96 \cdot 06^{\mathrm{a}, \mathrm{b}}$ & 2.41 & $96 \cdot 52^{\mathrm{a}}$ & 1.87 & 2.92 & 0.05 \\
\hline Glu & $82.29^{e}$ & 1.17 & $72.07^{f}$ & 1.42 & $93 \cdot 23^{c}$ & 0.82 & $94.67^{b}$ & 0.67 & $70 \cdot 89^{f}$ & 1.09 & $90.50^{\mathrm{d}}$ & 0.96 & $83.59^{e}$ & 0.74 & $96 \cdot 73^{\mathrm{a}}$ & 0.81 & $97.85^{\mathrm{a}}$ & 0.47 & 3.41 & 0.05 \\
\hline Gly & $95 \cdot 13^{\mathrm{a}}$ & 1.45 & $76 \cdot 62^{c}$ & 2.73 & $88 \cdot 90^{\mathrm{b}}$ & 2.09 & $88.07^{d}$ & 1.56 & $72 \cdot 34^{d}$ & 4.59 & $80 \cdot 05^{\mathrm{C}}$ & 3.32 & $76.90^{\mathrm{C}}$ & 1.97 & $89 \cdot 31^{b}$ & 3.55 & $96 \cdot 00^{\mathrm{a}}$ & 1.97 & 2.86 & 0.05 \\
\hline Arg & $92 \cdot 97^{\mathrm{a}, \mathrm{b}}$ & 1.26 & $86 \cdot 74^{d}$ & 1.67 & $91.76^{\mathrm{b}, \mathrm{c}}$ & 1.47 & $88.97^{b, c, d}$ & $1 \cdot 10$ & $85.40^{d}$ & 5.63 & $91 \cdot 27^{\mathrm{b}, \mathrm{c}}$ & 2.44 & $87.76^{\mathrm{c}, \mathrm{d}}$ & 1.65 & $95 \cdot 91^{\mathrm{a}}$ & 3.30 & $91.53^{\mathrm{b}, \mathrm{c}}$ & 1.69 & $1 \cdot 11$ & 0.05 \\
\hline Ala & $85 \cdot 49^{b}$ & 0.90 & $76 \cdot 68^{d}$ & 2.80 & $82 \cdot 29^{c}$ & 1.62 & $85 \cdot 56^{\mathrm{b}}$ & 1.74 & $77 \cdot 35^{d}$ & 1.37 & $90 \cdot 60^{\mathrm{a}}$ & 1.29 & $76.94^{d}$ & 1.99 & $90 \cdot 69^{a}$ & 0.92 & $89.94^{\mathrm{a}}$ & 2.39 & 1.97 & 0.05 \\
\hline Pro & $87.52^{b, c}$ & $2 \cdot 22$ & $78 \cdot 71^{d}$ & 3.60 & $85 \cdot 35^{c}$ & 2.49 & $91 \cdot 77^{\mathrm{a}}$ & 1.52 & $77 \cdot 73^{\mathrm{d}}$ & 1.85 & $93 \cdot 80^{\mathrm{a}}$ & 1.66 & $72 \cdot 74^{\mathrm{e}}$ & $3 \cdot 32$ & $90 \cdot 54^{\mathrm{a}, \mathrm{b}}$ & 0.86 & $86 \cdot 81^{\mathrm{C}}$ & 1.07 & $2 \cdot 38$ & 0.05 \\
\hline Cys & $68 \cdot 84^{\mathrm{d}, \mathrm{e}}$ & 1.51 & $62 \cdot 10^{f}$ & 1.49 & $82 \cdot 82^{c}$ & 1.07 & $93.33^{a}$ & 0.93 & $66 \cdot 25^{e, f}$ & 3.07 & $88 \cdot 84^{b}$ & 4.05 & $73 \cdot 13^{d}$ & 4.00 & $84 \cdot 62^{\mathrm{b}, \mathrm{c}}$ & $5 \cdot 30$ & $86.04^{\mathrm{b}, \mathrm{c}}$ & $2 \cdot 86$ & 3.69 & 0.05 \\
\hline Tyr & $78 \cdot 62^{\mathrm{c}}$ & 2.49 & $75 \cdot 31^{c}$ & 2.45 & $90 \cdot 16^{a}$ & 3.12 & $90.55^{a}$ & $2 \cdot 22$ & $83 \cdot 11^{\mathrm{b}}$ & 2.45 & $91.06^{\mathrm{a}}$ & 2.92 & $74.60^{\mathrm{C}}$ & 3.46 & $89.63^{\mathrm{a}}$ & 1.82 & $92 \cdot 95^{\mathrm{a}}$ & 2.69 & 2.43 & 0.05 \\
\hline Mean & $85 \cdot 51^{\mathrm{c}}$ & 0.36 & $75 \cdot 70^{d}$ & 1.93 & $87.84^{\mathrm{b}, \mathrm{c}}$ & 1.52 & $89.65^{a, b}$ & 1.21 & $75 \cdot 80^{d}$ & 1.98 & $90 \cdot 03^{\mathrm{a}, \mathrm{b}}$ & 2.03 & $78 \cdot 14^{d}$ & 1.90 & $91 \cdot 76^{\mathrm{a}}$ & 1.83 & $91.92^{\mathrm{a}}$ & 1.58 & $2 \cdot 26$ & 0.05 \\
\hline Total mean & $84.97^{c}$ & 0.75 & $76 \cdot 93^{d}$ & $2 \cdot 18$ & $88.01^{\mathrm{b}}$ & 1.57 & $88.54^{\mathrm{b}}$ & 1.49 & $77.98^{d}$ & 1.94 & $89.96^{\mathrm{b}}$ & 2.57 & $78 \cdot 12^{d}$ & $2 \cdot 32$ & $92 \cdot 81^{\mathrm{a}}$ & 1.98 & $93.32^{\mathrm{a}}$ & 2.01 & $2 \cdot 16$ & 0.05 \\
\hline
\end{tabular}

* TID values were calculated by correcting the values for apparent ileal digestibility for the basal endogenous losses. Values used for the basal endogenous losses were follows (g/kg of DM intake): Asp, 1.09; Ser, 0.83; Glu, 1.25; Gly, 1.24;

His, 0.26; Arg, 0.42; Thr, 0.73; Ala, 0.43; Pro, 0.76; Cys, 0.09; Tyr, 0.28; Val, 0.44; Met, 0.07; Lys, 0.40; Ile, 0.32; Leu, 0.55; Phe, 0.30; Trp, 0.08. 
Table 6. Mean true ileal digestibility concentrations ( $\mathrm{g} / \mathrm{kg} \mathrm{DM}$ ) for amino acid (AA) in brown rice, polished rice, buckwheat, oats, proso millet, foxtail millet, tartary buckwheat, adlay and whole wheat (Mean values with their standard errors)

\begin{tabular}{|c|c|c|c|c|c|c|c|c|c|c|c|c|c|c|c|c|c|c|c|c|}
\hline & \multicolumn{2}{|c|}{ Brown rice } & \multicolumn{2}{|c|}{ Polished rice } & \multicolumn{2}{|c|}{ Buckwheat } & \multicolumn{2}{|c|}{ Oats } & \multicolumn{2}{|c|}{ Proso millet } & \multicolumn{2}{|c|}{ Foxtail millet } & \multicolumn{2}{|c|}{ Tartary buckwheat } & \multicolumn{2}{|c|}{ Adlay } & \multicolumn{2}{|c|}{ Whole wheat } & \multirow[b]{2}{*}{ SEM } & \multirow[b]{2}{*}{$P$} \\
\hline & Mean & SEM & Mean & SEM & Mean & SEM & Mean & SEM & Mean & SEM & Mean & SEM & Mean & SEM & Mean & SEM & Mean & SEM & & \\
\hline \multicolumn{21}{|c|}{ Indispensable AA } \\
\hline His & $1.76^{\mathrm{C}}$ & 0.05 & $1.32^{\mathrm{e}}$ & 0.08 & $1.92^{\mathrm{b}}$ & 0.07 & $1.80^{\mathrm{b}, \mathrm{c}}$ & 0.09 & $1.78^{\mathrm{C}}$ & 0.09 & $1.77^{\mathrm{c}}$ & 0.08 & $1.60^{\mathrm{d}}$ & 0.08 & $1 \cdot 80^{\mathrm{b}, \mathrm{c}}$ & 0.07 & $2 \cdot 18^{\mathrm{a}}$ & 0.08 & 0.08 & 0.05 \\
\hline Thr & $2.45^{\mathrm{c}}$ & 0.09 & $1.71^{\mathrm{e}}$ & 0.08 & $2.97^{\mathrm{a}}$ & 0.07 & $2.56^{\mathrm{c}}$ & 0.10 & $2 \cdot 14^{\mathrm{d}}$ & 0.08 & $2 \cdot 82^{\mathrm{b}}$ & 0.08 & $2.43^{\mathrm{c}}$ & 0.09 & $2.56^{\mathrm{c}}$ & 0.09 & $2 \cdot 74^{\mathrm{b}}$ & 0.16 & 0.13 & 0.05 \\
\hline Val & $3.96^{\mathrm{d}}$ & 0.07 & $3.43^{9}$ & 0.08 & $4.02^{\mathrm{d}}$ & 0.08 & $4.54^{\mathrm{b}}$ & 0.09 & $4 \cdot 26^{\mathrm{c}}$ & 0.12 & $3.77^{e}$ & 0.08 & $3 \cdot 61^{f}$ & 0.10 & $5 \cdot 12^{\mathrm{a}}$ & 0.09 & $4 \cdot 26^{\mathrm{c}}$ & 0.08 & 0.17 & 0.05 \\
\hline Met & $0.55^{f}$ & 0.01 & $0.95^{\mathrm{d}}$ & 0.08 & $1.05^{\mathrm{d}}$ & 0.01 & $0.58^{f}$ & 0.01 & $1.47^{\mathrm{c}}$ & 0.03 & $2 \cdot 70^{\mathrm{a}}$ & 0.14 & $0.71^{\mathrm{e}}$ & 0.08 & $1.90^{\mathrm{b}}$ & 0.08 & $1.03^{\mathrm{d}}$ & 0.02 & 0.23 & 0.05 \\
\hline Lys & $2 \cdot 16^{\mathrm{d}}$ & 0.08 & $1.82^{\mathrm{e}}$ & 0.07 & $4 \cdot 26^{\mathrm{a}}$ & 0.08 & $2.56^{\mathrm{c}}$ & 0.08 & $0.44^{i}$ & 0.02 & $0.62^{\mathrm{h}}$ & 0.08 & $3.25^{\mathrm{b}}$ & 0.09 & $0.84^{\mathrm{g}}$ & 0.03 & $1.27^{f}$ & 0.03 & 0.43 & 0.05 \\
\hline Ile & $2 \cdot 56^{f}$ & 0.12 & $2 \cdot 25^{\mathrm{g}}$ & 0.08 & $2.98^{\mathrm{d}}$ & 0.08 & $3.40^{\mathrm{c}}$ & 0.08 & $3.53^{b, c}$ & 0.08 & $3.55^{\mathrm{b}}$ & 0.08 & $2.83^{\mathrm{e}}$ & 0.10 & $3.80^{\mathrm{a}}$ & 0.09 & $3 \cdot 51^{b, c}$ & 0.08 & 0.18 & 0.05 \\
\hline Leu & $5 \cdot 70^{f}$ & 0.11 & $4.77^{\mathrm{h}}$ & 0.08 & $5 \cdot 77^{f}$ & 0.07 & $7.35^{\mathrm{d}}$ & 0.08 & $12 \cdot 63^{\mathrm{b}}$ & 0.21 & $12 \cdot 35^{\mathrm{c}}$ & 0.16 & $5.00^{9}$ & 0.09 & $15 \cdot 01^{a}$ & 0.16 & $7 \cdot 10^{\mathrm{e}}$ & 0.16 & 1.29 & 0.05 \\
\hline Phe & $3 \cdot 61^{\dagger}$ & 0.09 & $3.20^{9}$ & 0.07 & $4 \cdot 16^{\mathrm{e}}$ & 0.08 & $4.99^{\mathrm{C}}$ & 0.09 & $5 \cdot 43^{\mathrm{a}}$ & 0.08 & $5 \cdot 21^{b}$ & 0.15 & $3.56^{\dagger}$ & 0.09 & $5 \cdot 45^{a}$ & 0.06 & $4.80^{d}$ & 0.06 & 0.29 & 0.05 \\
\hline Trp & $1.69^{\mathrm{a}}$ & 0.03 & $0.62^{\mathrm{h}}$ & 0.01 & $0.96^{\mathrm{e}}$ & 0.01 & $1.09^{d}$ & 0.02 & $0.74^{\mathrm{g}}$ & 0.03 & $1.62^{\mathrm{b}}$ & 0.03 & $1 \cdot 14^{\mathrm{c}}$ & 0.03 & $0.87^{f}$ & 0.03 & $0.91^{f}$ & 0.04 & 0.12 & 0.05 \\
\hline Total & $24.44^{f}$ & 0.40 & $20 \cdot 06^{g}$ & 0.55 & $28 \cdot 10^{\mathrm{e}}$ & 0.55 & $28.86^{d}$ & 0.53 & $32 \cdot 41^{\mathrm{c}}$ & 0.53 & $34.42^{b}$ & 0.74 & $24 \cdot 15^{\dagger}$ & 0.68 & $37.36^{a}$ & 0.59 & $27.78^{\mathrm{e}}$ & 0.62 & 1.81 & 0.05 \\
\hline \multicolumn{21}{|c|}{ Dispensable AA } \\
\hline Asp & $6.35^{\mathrm{c}}$ & 0.12 & $5 \cdot 17^{f}$ & 0.17 & $8 \cdot 11^{\mathrm{a}}$ & 0.12 & $7.07^{b}$ & 0.10 & $4.58^{\mathrm{g}}$ & 0.09 & $5.89^{\mathrm{e}}$ & 0.15 & $6 \cdot 31^{\mathrm{c}, \mathrm{d}}$ & 0.11 & $6 \cdot 17^{d}$ & 0.08 & $4 \cdot 65^{\mathrm{g}}$ & 0.09 & 0.38 & 0.05 \\
\hline Ser & $3.68^{\mathrm{e}}$ & 0.09 & $2 \cdot 86^{9}$ & 0.15 & $4.03^{d}$ & 0.06 & $4.37^{c}$ & 0.11 & $5 \cdot 32^{a}$ & 0.10 & $3.99^{d}$ & 0.09 & $3.47^{\dagger}$ & 0.10 & $4.63^{\mathrm{b}}$ & 0.12 & $4.77^{\mathrm{b}}$ & 0.09 & 0.25 & 0.05 \\
\hline Glu & $11.95^{\dagger}$ & 0.17 & $10.09^{h}$ & 0.20 & $15.89^{e}$ & 0.14 & $21 \cdot 17^{c}$ & 0.15 & $17 \cdot 81^{d}$ & 0.27 & $17.66^{d}$ & 0.19 & $11.30^{\mathrm{g}}$ & 0.10 & $23.79^{\mathrm{b}}$ & 0.20 & $30.09^{a}$ & 0.15 & $2 \cdot 17$ & 0.05 \\
\hline Gly & $3 \cdot 61^{\mathrm{b}}$ & 0.05 & $2.53^{\mathrm{e}}$ & 0.09 & $4 \cdot 83^{a}$ & 0.11 & $4.54^{\mathrm{b}}$ & 0.08 & $1.35^{\mathrm{h}}$ & 0.09 & $1.91^{9}$ & 0.08 & $3.61^{d}$ & 0.09 & $2 \cdot 18^{\dagger}$ & 0.09 & $4.25^{\mathrm{c}}$ & 0.09 & 0.42 & 0.05 \\
\hline Arg & $6 \cdot 76^{\mathrm{b}}$ & 0.09 & $5 \cdot 24^{d}$ & 0.10 & $9.00^{\mathrm{a}}$ & 0.14 & $6 \cdot 37^{\mathrm{c}}$ & 0.08 & $1.26^{\mathrm{h}}$ & 0.08 & $3.03^{g}$ & 0.08 & $6 \cdot 69^{\mathrm{b}}$ & 0.13 & $4.05^{\dagger}$ & 0.14 & $4.84^{e}$ & 0.09 & 0.76 & 0.05 \\
\hline Ala & $4.02^{\mathrm{C}}$ & 0.04 & $3 \cdot 36^{\mathrm{d}}$ & 0.12 & $3.19^{e}$ & 0.06 & $4.04^{\mathrm{C}}$ & 0.08 & $9.43^{\mathrm{a}}$ & 0.17 & $7.62^{\mathrm{b}}$ & 0.11 & $2.95^{f}$ & 0.08 & $9.35^{\mathrm{a}}$ & 0.09 & $3 \cdot 34^{d}$ & 0.09 & 0.91 & 0.05 \\
\hline Pro & $3.60^{f}$ & 0.09 & $2 \cdot 77^{\mathrm{h}}$ & 0.13 & $3 \cdot 14^{\mathrm{g}}$ & 0.09 & $5.35^{\mathrm{e}}$ & 0.09 & $6 \cdot 71^{d}$ & 0.16 & $7.41^{\mathrm{C}}$ & 0.13 & $2 \cdot 33^{i}$ & 0.11 & $8.07^{b}$ & 0.08 & $10.02^{\mathrm{a}}$ & 0.12 & 0.90 & 0.05 \\
\hline Cys & $0.43^{e, f}$ & 0.01 & $0.39^{9}$ & 0.01 & $0.93^{\mathrm{b}}$ & 0.01 & $1.32^{\mathrm{a}}$ & 0.01 & $0 \cdot 40^{\mathrm{f}, \mathrm{g}}$ & 0.02 & $0.61^{\mathrm{C}}$ & 0.03 & $0.45^{\mathrm{e}}$ & 0.02 & $0.50^{d}$ & 0.03 & $0.91^{\mathrm{b}}$ & 0.03 & 0.11 & 0.05 \\
\hline Tyr & $2 \cdot 80^{\mathrm{e}}$ & 0.09 & $2 \cdot 54^{\dagger}$ & 0.08 & $2.33^{\mathrm{g}}$ & 0.08 & $3 \cdot 28^{c}$ & 0.08 & $3 \cdot 63^{\mathrm{b}}$ & 0.11 & $3.08^{d}$ & 0.10 & $1 \cdot 81^{\mathrm{h}}$ & 0.08 & $3 \cdot 91^{\mathrm{a}}$ & 0.08 & $3.01^{d}$ & 0.09 & 0.22 & 0.05 \\
\hline Total & $43.20^{\mathrm{e}}$ & 0.20 & $34.93^{9}$ & 0.57 & $51.45^{\mathrm{d}}$ & 0.75 & $57.52^{\mathrm{c}}$ & 0.65 & $50 \cdot 49^{d}$ & 0.83 & $51 \cdot 20^{d}$ & 0.74 & $38.92^{f}$ & 0.69 & $62 \cdot 64^{b}$ & 0.72 & $65.88^{a}$ & 0.71 & 3.46 & 0.05 \\
\hline Total AA & $67 \cdot 70^{f}$ & 0.58 & $54.99^{\text {h }}$ & $1 \cdot 12$ & $79.55^{e}$ & 1.25 & $86 \cdot 38^{c}$ & $1 \cdot 17$ & $82.90^{d}$ & 1.31 & $85 \cdot 62^{c}$ & 1.48 & $63.07^{9}$ & 1.36 & $100 \cdot 00^{\mathrm{a}}$ & 1.31 & $93 \cdot 66^{\mathrm{b}}$ & 1.32 & 3.52 & 0.05 \\
\hline
\end{tabular}

a,b,c,d,e,f,g,h Mean values within a row with unlike superscript letters were significantly different $(P<0.05)$. 
Asia and Africa ${ }^{(32)}$. Adlay (Coix lachryma-jobi L.) is mainly cultivated in China and Japan ${ }^{(33)}$. Many recent studies indicated that the consumption of these cereal grains are beneficial because they reduce the risk of acquiring chronic diseases ${ }^{(30,32,34-36)}$.

The protein and AA contents of protein sources should be determined and the TID of each indispensable AA in the test protein should be used to allow calculation of accurate DIAAS values ${ }^{(16)}$. Grain proteins play many important roles in human health; thus, assessing their quality after processing is important. A few decades ago, the FAO established a method for protein nutritional value assessment. AA digestibility determination at the terminal ileum is more accurate than the traditional faecal method $^{(37)}$. Although ileal digestibility may not be a perfect measure to determine net AA absorption, it is considerably closer than the AA digestibility determined over the total digestive tract ${ }^{(38)}$. TID values are usually very accurate unless a protein has been overheated, which may result in reduced digestibility of Lys ${ }^{(15)}$. The variations in the AID values may be a result of the differences among grain varieties and growing conditions of the grains ${ }^{(39)}$. Therefore, protein evaluation can be improved by calculating the TID values of $\mathrm{AA}$ and removing the influences of basal endogenous losses of AA on determined digestibility values ${ }^{(6)}$.

In the 2011 Protein Quality Expert Consultation, DIAAS was reported to provide more accurate protein quality scores than the PDCAAS $^{(15)}$. However, nearly all available DIAAS data were obtained from pig models, and those derived from humans remains insufficient ${ }^{(11)}$. In this study, the DIAAS values obtained from polished rice, oats and whole wheat were lower than those reported by Cervantes-Pahm et al. ${ }^{(28)}$, Mathai et al..$^{(40)}$ and Abelilla et $a l .{ }^{(41)}$. According to the DIAAS cut-off value introduced by an FAO Expert Consultation report and the study performed by Cervantes-Pahm et al. ${ }^{(15,28)}$, only dehulled oats are good protein sources for human consumption because its DIAAS is 77. However, the DIAAS was 68 for buckwheat, 47 for tartary buckwheat and 43 for oats in this study. It is possible that buckwheat and tartary buckwheat are better protein sources than oats. However, further work is needed to compare the digestibility in the rat-based assay to that in human-based studies with the use of the same foods when consumed by humans.

In conclusion, diets based on proso millet and foxtail millet require more AA supplementation than those based on buckwheat, tartary buckwheat, oats and brown rice for them to meet the balanced AA based on DIAAS values in this study. DIAAS value obtained from cereal grains can provide comprehensive nutritional information and a scientific basis for the evaluation of the nutritional values of proteins contained in different cereals. Given the DIAAS values obtained from cereal grains, the rational combination of various cereal grains had increased protein quality in human diets and is useful as a scientific basis for formulating balanced diets.

\section{Acknowledgements}

The authors thank Shane M. Rutherfurd at Massey University for his skilful technical assistance. The authors also thank Professor Paul J. Moughan at Massey University for his critical reading of this manuscript and helpful suggestions. 
This study was financially supported by the Special Funds of Basic Research of Central Public Welfare Institute (no. ZX1731) and the non-profit industry (grain) Scientific Research Special Fund Agreement (no. 201513003-8) from the Ministry of Finance, People's Republic of China.

F. H. was involved in the design of the experimental protocol and discussion of the results. F. L. H., Y. W., L. P. F., G. S., X. C., P. J., H. J. M. and Y. Y. H. performed the experiments and collected data. Y. W. and F. L. H. wrote the first draft of the manuscript; and all authors critically reviewed the manuscript and approved the final content.

The authors declare that there are no conflicts of interest.

\section{References}

1. Moughan PJ (2012) Dietary protein for human health (Preface). Br J Nutr 108, S1-S2.

2. Food and Agriculture Organization of the United Nations (1991) Protein Quality Evaluation. Report of the Joint FAO/ WHO Expert Consultation, Bethesda, Md., USA, 4-8 December 1989. FAO Food and Nutrition Paper 51. Rome: FAO.

3. Rutherfurd SM, Fanning AC, Miller BJ, et al. (2015) Protein digestibility-corrected amino acid scores and digestible indispensable amino acid scores differentially describe protein quality in growing male rats. J Nutr 145, 372-379.

4. Schaafsma G (2012) Advantages and limitations of the protein digestibility-corrected amino acid score (PDCAAS) as a method for evaluating protein quality in human diets. $\mathrm{Br} J$ Nutr 108, S333-S336.

5. Moughan PJ \& Stevens BR (2012) Digestion and absorption of protein. In Biochemical, Physiological and Molecular Aspects of Human Nutrition, pp. 162-178 [MH Stipanuk and MA Caudill, editors]. St Louis, MO: Elsevier.

6. Stein HH, Seve B, Fuller MF, et al. (2007) Invited review: amino acid bioavailability and digestibility in pig feed ingredients: terminology and application. J Anim Sci 85, 172-180.

7. Moughan PJ (2003) Amino acid availability: aspects of chemical analysis and bioassay methodology. Nutr Res Rev 16, 127-141.

8. Rowan AM, Moughan PJ, Wilson MN, et al. (1994) Comparison of the ileal and fecal digestibility of dietary amino-acids in adult humans and evaluation of the pig as a model animal for digestion studies in man. Br J Nutr 71, 29-42.

9. Schaafsma G (2000) The protein digestibility-corrected amino acid score. J Nutr 130, 1865S-1867S.

10. Schaafsma G (2005) The protein digestibility-corrected amino acid score (PDCAAS) - a concept for describing protein quality in foods and food ingredients: a critical review. J $A O A C$ Int 88, 988-994.

11. Lee WTK, Weisell R, Albert J, et al. (2016) Research approaches and methods for evaluating the protein quality of human foods proposed by an FAO expert working group in 2014. J Nutr 146, 929-932.

12. Boye J, Wijesinha-Bettoni R \& Burlingame B (2012) Protein quality evaluation twenty years after the introduction of the protein digestibility corrected amino acid score method. Br J Nutr 108, S183-S211.

13. Millward DJ, Layman DK, Tome D, et al. (2008) Protein quality assessment: impact of expanding understanding of protein and amino acid needs for optimal health. Am J Clin Nutr 87, 1576S-1581S.

14. Gilani GS, Xiao CW \& Cockell KA (2012) Impact of antinutritional factors in food proteins on the digestibility of protein and the bioavailability of amino acids and on protein quality. Br J Nutr 108, S315-S332.

15. Food and Agriculture Organization of the United Nations (2013) Dietary protein quality evaluation in human nutrition. Report of an FAO Expert Consultation. FAO Food and Nutrition Paper 92. http://www.fao.org/ag/humannutrition/3597802317b979a686a57aa4593304ff c17f06.pdf

16. Wolfe RR, Rutherfurd SM, Kim IY, et al. (2016) Protein quality as determined by the digestible indispensable amino acid score: evaluation of factors underlying the calculation. Nutr Rev 74, 584-599.

17. Bwibo NO \& Neumann CG (2003) The need for animal source foods by Kenyan children. J Nutr 133, 3936S-3940S.

18. Deglaire A \& Moughan PJ (2012) Animal models for determining amino acid digestibility in humans - a review. $\mathrm{Br} J$ Nutr 108, S273-S281.

19. Butts CA, Monro JA \& Moughan PJ (2012) In vitro determination of dietary protein and amino acid digestibility for humans. Br J Nutr 108, S282-S287.

20. Food and Agriculture Organization of the United Nations (2014) Research Approaches and Methods for Evaluating the Protein Quality of Human Foods: Report of a FAO Expert Working Group. Rome: FAO.

21. Moughan PJ \& Rutherfurd SM (2012) Gut luminal endogenous protein: implications for the determination of ileal amino acid digestibility in humans. Br J Nutr 108, S258-S263.

22. Rutherfurd SM \& Moughan PJ (2003) The rat as a model animal for the growing pig in determining ileal amino acid digestibility in soya and milk proteins. J Anim Physiol Anim Nutr 87, 292-300.

23. Rutherfurd SM \& Moughan PJ (1998) The digestible amino acid composition of several milk proteins: application of a new bioassay. J Dairy Sci 81, 909-917.

24. Rutherfurd SM \& Gilani GS (2009) Amino acid analysis. Curr Protoc Protein Sci 58, 11.9.1-11.9.37.

25. Short FJ, Gorton P, Wiseman J, et al. (1996) Determination of titanium dioxide added as an inert marker in chicken digestibility studies. Anim Feed Sci Technol 59, 215-221.

26. Rutherfurd SM, Bains K \& Moughan PJ (2012) Available lysine and digestible amino acid contents of proteinaceous foods of India. Br J Nutr 108, S59-S68.

27. Rutherfurd SM, Cui J, Goroncy AK, et al. (2015) Dietary protein structure affects endogenous ileal amino acids but not true ileal amino acid digestibility in growing male rats. $J$ Nutr 145, 193-198.

28. Cervantes-Pahm SK, Liu Y \& Stein HH (2014) Digestible indispensable amino acid score and digestible amino acids in eight cereal grains. Br J Nutr 111, 1663-1672.

29. Charan J \& Kantharia ND (2013) How to calculate sample size in animal studies? J Pharmacol Pharmacother 4, 303-306.

30. Zhang Z, Zhou M, Tang Y, et al. (2012) Bioactive compounds in functional buckwheat food. Food Res Int 49, 389-395.

31. Lu H, Zhang J, Liu K, et al. (2009) Earliest domestication of common millet (Panicum miliaceum) in East Asia extended to 10000 years ago. Proc Natl Acad Sci U S A 106, 7367-7372.

32. Amadou I, Le G, Amza T, et al. (2013) Purification and characterization of foxtail millet-derived peptides with antioxidant and antimicrobial activities. Food Res Int 51, 422-428.

33. Wang L, Chen C, Su A, et al. (2016) Structural characterization of phenolic compounds and antioxidant activity of the phenolic-rich fraction from defatted adlay (Coix lachrymajobi L. var. ma-yuen Stapf) seed meal. Food Chem 196, 509-517.

34. Guo X, Ma Y, Parry J, et al. (2011) Phenolics content and antioxidant activity of tartary buckwheat from different locations. Molecules 16, 9850-9867. 
35. Zhang L, Liu R \& Niu W (2014) Phytochemical and antiproliferative activity of proso millet. PLOS ONE 9 , e104058.

36. Chen H, Chung C, Chiang W, et al. (2011) Anti-inflammatory effects and chemical study of a flavonoid-enriched fraction from adlay bran. Food Chem 126, 1741-1748.

37. Wielen NVD, Moughan PJ \& Mensink M (2017) Amino acid absorption in the large intestine of humans and porcine models. J Nutr 147, 1493-1498.

38. Fuller M (2012) Determination of protein and amino acid digestibility in foods including implications of gut microbial amino acid synthesis. BrJ Nutr 108, S238-S246.
39. Nosworthy MG, Neufeld J, Frohlich P, et al. (2017) Determination of the protein quality of cooked Canadian pulses. Food Sci Nutr 5, 896-903.

40. Mathai JK, Liu Y \& Stein HH (2017) Values for digestible indispensable amino acid scores (DIAAS) for some dairy and plant proteins may better describe protein quality than values calculated using the concept for protein digestibility-corrected amino acid scores (PDCAAS). Br J Nutr 117, 490-499.

41. Abelilla JJ, Liu Y \& Stein HH (2018) Digestible indispensable amino acid score (DIAAS) and protein digestibility corrected amino acid score (PDCAAS) in oat protein concentrate measured in 20 to 30 kilogram pigs. J Sci Food Agric 98, 410-414. 